\title{
Global Existence of Large BV Solutions in a Model of Granular Flow
}

\author{
Debora Amadori* and Wen Shen** \\ (*): Dipartimento di Matematica Pura ed Applicata, University of L'Aquila, Italy. \\ Email: amadori@univaq.it \\ $(* *)$ : Department of Mathematics, Penn State University, U.S.A.. \\ Email: shen_w@math.psu.edu
}

January 5, 2009

\begin{abstract}
In this paper we analyze a set of equations proposed by Hadeler and Kuttler [20], describing the flow of granular matter in terms of the heights of a standing layer and of a moving layer. By a suitable change of variables, the system can be written as a $2 \times 2$ hyperbolic system of balance laws, which we study in the one-dimensional case. The system is linearly degenerate along two straight lines in the phase plane, and therefore is weakly linearly degenerate at the point of the intersection. The source term is quadratic, consisting of product of two quantities, which are transported with strictly different speeds. Assuming that the initial height of the moving layer is sufficiently small, we prove the global existence of entropy-weak solutions to the Cauchy problem, for a class of initial data with bounded but possibly large total variation.
\end{abstract}

\section{Introduction and main results}

We consider a model for the flow of a granular material, such as sand or gravel, which was proposed in [20]. The material is divided in two parts: a moving layer on top and a standing layer at the bottom. We denote by $h$ the thickness of the moving layer, and by $u$ the height of the standing layer. The evolution of these two quantities is described by the system

$$
\left\{\begin{array}{l}
h_{t}=\operatorname{div}(h \nabla u)-(1-|\nabla u|) h \\
u_{t}=(1-|\nabla u|) h
\end{array}\right.
$$

According to (1.1), the moving layer slides downhill, in the direction of steepest descent, with speed proportional to the slope of the standing layer. There is a critical slope, which in this normalized model is $|\nabla u|=1$, with the following property. If $|\nabla u|>1$ then grains initially at rest are hit by rolling grains of the moving layer and start moving as well. Hence the moving layer gets bigger. On the other hand, if $|\nabla u|<1$, grains which are rolling can be deposited on the bed. Hence the moving layer becomes smaller.

Throughout the following, we study the case of one space dimension. We assume $u_{x} \geq 0$ and drop absolute values. Differentiating the second equation in (1.1) in the space variable $x$ and 
setting $p=u_{x}$, one obtains the system of balance laws

$$
\left\{\begin{aligned}
h_{t}-(h p)_{x} & =(p-1) h \\
p_{t}+((p-1) h)_{x} & =0
\end{aligned}\right.
$$

Here $h \geq 0$ and $p \geq 0$. A direct computation shows that, except at the point $(p, h)=(0,0)$, this system is strictly hyperbolic.

For a suitable class of initial data, in [26] one of the authors established the global existence of smooth solutions. However, with more general initial data, due to the nonlinearity of the flux, the solution will develop discontinuities (shocks) in finite time. The purpose of the present paper is to prove the global existence of BV solutions, for a class of initial data with bounded but possibly large total variation.

More precisely, consider initial data of the form

$$
h(0, x)=\bar{h}(x), \quad p(0, x)=\bar{p}(x) .
$$

We assume that $\bar{h}: \mathbb{R} \mapsto \mathbb{R}_{+}^{*}$ and $\bar{p}: \mathbb{R} \mapsto \mathbb{R}_{+}^{*}$ are non-negative functions with bounded variation, such that

$$
\begin{array}{rlrl}
\text { Tot.Var. }\{\bar{h}\} & \leq M, & & \text { Tot.Var. }\{\bar{p}\} \leq M, \\
\|\bar{h}\|_{\mathbf{L}^{1}} & \leq M, & \|\bar{p}-1\|_{\mathbf{L}^{1}} \leq M,
\end{array}
$$

and

$$
\bar{p}(x) \geq p_{0}>0,
$$

for some constants $M$ (possibly large) and $p_{0}$. Our main result is the following:

Theorem 1 (global existence of large $B V$ solutions for the Cauchy problem). For any constants $M, p_{0}>0$, there exists $\delta>0$ small enough such that, if (1.4)-(1.6) hold together with

$$
\|\bar{h}\|_{\mathbf{L}^{\infty}} \leq \delta,
$$

then the Cauchy problem (1.2)-(1.3) has an entropy weak solution, defined for all $t \geq 0$, with uniformly bounded total variation.

Compared with previous literature, the main novelty of the present result stems from the fact that

i. The system (1.2) contains source terms;

ii. We are assuming a small $\mathbf{L}^{\infty}$ bound on $h(\cdot)$, but not on the other component $p(\cdot)$;

iii. We have arbitrarily large BV data.

In addition, we observe that the system (1.2) is strictly hyperbolic, but one of the characteristic fields is neither genuinely nonlinear nor linearly degenerate. This requires additional care throughout the analysis.

We recall that, for systems without source terms and small BV data, the global existence and uniqueness of entropy-weak solutions to the Cauchy problem is well known. Even in the general case of systems which are neither genuinely nonlinear nor linearly degenerate, global solutions 
have been constructed by the Glimm scheme [18, 21, 23, 27], by front tracking approximations $[4,5]$, and by vanishing viscosity approximations [7]. In some special cases, existence and uniqueness of global solutions in the presence of a source term were proved in $[16,22,12]$ and in $[2,15,1,13,14]$, respectively.

Global existence of solutions to hyperbolic systems with large BV data is a more difficult, still largely open problem. In addition to the special system [24], two main cases are known in the literature, where global existence of large BV solutions is achieved. One is the case of Temple class systems [25]. Here one can measure the wave strengths in terms of Riemann invariants, so that the total strength of all wave fronts does not increase in time, across each interaction.

A second major result [19] refers to general $2 \times 2$ systems, where again we can measure wave strengths in terms of Riemann coordinates. To see what happens at an interaction, let $\sigma_{1}$ and $\sigma_{2}$ be the strengths of the incoming waves of different families, and let $\sigma_{1}^{\prime}$ and $\sigma_{2}^{\prime}$ be the strengths of the outgoing waves. One then has a cubic interaction estimate of the form

$$
\left|\sigma_{1}^{\prime}-\sigma_{1}\right|+\left|\sigma_{2}^{\prime}-\sigma_{2}\right|=\mathcal{O}(1) \cdot\left|\sigma_{1}\right| \cdot\left|\sigma_{2}\right| \cdot\left(\left|\sigma_{1}\right|+\left|\sigma_{2}\right|\right) .
$$

Thanks to the last term on the right hand side, if the $\mathbf{L}^{\infty}$ norm of the solution is sufficiently small, the increase of total variation produced by the interaction is very small, and a global existence result of large BV solutions can again be established.

We remark that the cubic estimate in (1.8) is useless in our case, since the $\mathbf{L}^{\infty}$ norm of the component $p$ in (1.2) can be large, and so is the additional term $\left|\sigma_{1}\right|+\left|\sigma_{2}\right|$ in (1.8).

The validity of Theorem 1 relies heavily on some special properties of the hyperbolic system (1.2), namely

- It is linearly degenerate along the straight line where $h=0$. In the region where $h$ is very small, the second field of the system is "almost Temple class": the rarefaction curve and the shock curve of the $2^{\text {nd }}$ family through the same point are almost straight lines and are very close to each other, see Lemma 2 in Section 3. This allows us to replace the estimate (1.8) with

$$
\left|\sigma_{1}^{\prime}-\sigma_{1}\right|+\left|\sigma_{2}^{\prime}-\sigma_{2}\right|=\mathcal{O}(1) \cdot\left|\sigma_{1}\right| \cdot\left|\sigma_{2}\right| \cdot\|h\|_{\mathbf{L}^{\infty}} .
$$

- The source term involves the quadratic form $h(p-1)$. Here the quantities $h$ and $p-1$ have large, but bounded $\mathbf{L}^{1}$ norms. Moreover, they are transported with strictly different speeds. The total strength of the source term is thus expected to be $\mathcal{O}(1) \cdot\|h\|_{\mathbf{L}^{1}} \cdot\|p-1\|_{\mathbf{L}^{1}}$. In addition, since $h$ itself is a factor in the source term, one can obtain a uniform bound on the norm $\|h\|_{\mathbf{L}^{\infty}}$, valid for all times $t \geq 0$.

The remainder of the paper is organized as follows. In Section 2 we analyze the geometry of shock and rarefaction curves of the system. In Section 3 we prove the interaction estimates for our system for small $\|h\|_{\mathbf{L}} \infty$. In Section 4, ignoring the effect of wave interactions, we prove all the a priori estimates needed. Namely, we prove an $\mathbf{L}^{1}$ bound on $p-1$ and $h$, a global lower bound on $p$, the $\mathbf{L}^{\infty}$ bounds on $h$ and $p$, and eventually the BV bounds on $h$ and $p$. In Section 5 we put all the a priori estimates together and prove Theorem 1. Concluding remarks are given at the end of the paper, in Section 6.

For a derivation of the model (1.1) of granular flow we refer to [20]. Other models can be found in [6]. A mathematical analysis of steady state solutions for (1.1) was carried out in $[10,11]$. See also the interesting book [17]. Note that, besides [26], the present paper provides the first analytical study of time dependent solutions to this system. 


\section{Analysis of the Riemann problem; Geometry of shock and rarefaction curves}

We begin by analyzing the geometry of wave curves, in particular shock and rarefaction curves

for the Riemann problem. Writing the the system of balance laws (1.2) in quasilinear form, the corresponding Jacobian matrix is computed as

$$
A(h, p)=\left(\begin{array}{cc}
-p & -h \\
p-1 & h
\end{array}\right) .
$$

The characteristic polynomial is

$$
\lambda^{2}-(h-p) \lambda-h .
$$

If $(p-h)^{2}+4 h>0$ (which holds except at $(h, p)=(0,0)$ ), this polynomial has real distinct roots $\lambda_{1}<\lambda_{2}$, namely

$$
\lambda_{1,2}=\frac{1}{2}\left[h-p \pm \sqrt{(p-h)^{2}+4 h}\right] .
$$

Since we are interested in the case $h \geq 0, p \geq 0$, we consider the domain

$$
\Omega=\{(h, p): h \geq 0, p>0\},
$$

on which the system (1.2) is strictly hyperbolic. The corresponding eigenvectors have the form

$$
r_{1}(h, p)=\left(\begin{array}{c}
1 \\
-\frac{\lambda_{1}+1}{\lambda_{1}}
\end{array}\right), \quad r_{2}(h, p)=\left(\begin{array}{c}
-\frac{\lambda_{2}}{\lambda_{2}+1} \\
1
\end{array}\right) .
$$

For $p=1$ we have

$$
\lambda_{1}=-1, \quad \lambda_{2}=h, \quad r_{1}=\left(\begin{array}{l}
1 \\
0
\end{array}\right), \quad r_{2}=\left(\begin{array}{c}
\frac{-h}{1+h} \\
1
\end{array}\right)
$$

and for $h=0$ we have

$$
\lambda_{1}=-p, \quad \lambda_{2}=0, \quad r_{1}=\left(\begin{array}{c}
1 \\
\frac{1-p}{p}
\end{array}\right), \quad r_{2}=\left(\begin{array}{l}
0 \\
1
\end{array}\right) .
$$

When $h$ is small, i.e., with $h \approx 0$, we have

$$
\lambda_{1,2}=\frac{1}{2}\left(h-p \pm\left[p-h+\frac{2 h}{p}+\mathcal{O}\left(h^{2}\right)\right]\right),
$$

therefore the following expansions hold

$$
\lambda_{1}=-p+\frac{p-1}{p} h+\mathcal{O}\left(h^{2}\right), \quad \lambda_{2}=\frac{h}{p}+\mathcal{O}\left(h^{2}\right) .
$$


Note that for $p-h>0$ we have the following estimate for the 1-characteristic speed:

$$
\lambda_{1}=\frac{1}{2}\left[-(p-h)-\sqrt{(p-h)^{2}+4 h}\right] \leq-(p-h)<0 .
$$

By the identities $\lambda_{1} \lambda_{2}=-h$ and $\lambda_{1}+\lambda_{2}=h-p$, we get

$$
\partial_{h} \lambda_{1}=-\frac{1+\lambda_{1}}{\lambda_{2}-\lambda_{1}}, \quad \partial_{p} \lambda_{1}=\frac{\lambda_{1}}{\lambda_{2}-\lambda_{1}}, \quad \partial_{h} \lambda_{2}=\frac{1+\lambda_{2}}{\lambda_{2}-\lambda_{1}}, \quad \partial_{p} \lambda_{2}=-\frac{\lambda_{2}}{\lambda_{2}-\lambda_{1}},
$$

and the directional derivatives

$$
r_{1} \bullet \lambda_{1}=-\frac{2\left(\lambda_{1}+1\right)}{\lambda_{2}-\lambda_{1}} \approx \frac{2(p-1)}{p}, \quad r_{2} \bullet \lambda_{2}=-\frac{2 \lambda_{2}}{\lambda_{2}-\lambda_{1}} \approx-2 \frac{h}{p^{2}} .
$$

We see that the system is weakly linearly degenerate at the point $(h, p)=(0,1)$. The first characteristic field is genuinely nonlinear away from the line $p=1$ and the second field is genuinely nonlinear away from the line $h=0$. See Figure 1 for the characteristic curves. A result on the global existence of smooth solutions of the system (1.2) was recently proved in [26].

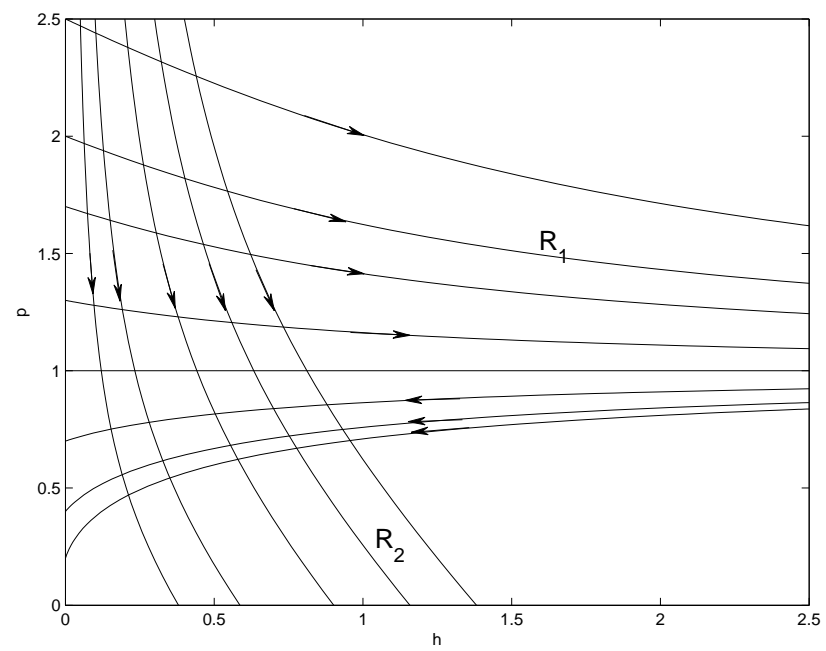

Figure 1: Characteristic curves of the two families in the $h-p$ plane. The arrows point in the direction of increasing eigenvalues.

To complete the preliminary analysis on the system (1.2), we derive the equations for the shocks curves. Let $\left(h_{l}, p_{l}\right)$ be the left state of a shock. The point $(h, p)$ that can be connected to $\left(h_{l}, p_{l}\right)$ by a shock with shock speed $s$ satisfies the Rankine-Hugoniot conditions

$$
-\left(h p-h_{l} p_{l}\right)=s\left(h-h_{l}\right), \quad\left(h p-h_{l} p_{l}\right)-\left(h-h_{l}\right)=s\left(p-p_{l}\right),
$$

which gives

$$
(s+1)\left(h-h_{l}\right)+s\left(p-p_{l}\right)=0, \quad s=-\frac{h-h_{l}}{h+p-h_{l}-p_{l}} .
$$

Using the identities

$$
h p-h_{l} p_{l}=h\left(p-p_{l}\right)+p_{l}\left(h-h_{l}\right)=h_{l}\left(p-p_{l}\right)+p\left(h-h_{l}\right),
$$


from (2.8) we get

$$
s^{2}-s\left(h-p_{l}\right)-h=0 \quad \text { and } \quad s^{2}-s\left(h_{l}-p\right)-h_{l}=0,
$$

these are both characteristic polynomials.

Hence, the shock curve of the $1^{s t}$ family with left state $\left(h_{l}, p_{l}\right)$, parametrized by $h$, is given by

$$
p-p_{l}=-\frac{s_{1}+1}{s_{1}}\left(h-h_{l}\right), \quad s_{1}=\lambda_{1}\left(h, p_{l}\right) .
$$

One can easily verify that the Liu admissibility condition is satisfied if $\left(h-h_{l}\right)\left(p_{l}-1\right)<0$, that is, $s_{1}(h) \leq s_{1}(\eta) \leq s_{1}\left(h_{l}\right)$ for all $\eta$ between $h$ and $h_{l}$.

Similarly, the shock curve of the $2^{\text {nd }}$ family with left state $\left(h_{l}, p_{l}\right)$, parametrized by $p$, is given by

$$
h-h_{l}=-\frac{s_{2}}{s_{2}+1}\left(p-p_{l}\right), \quad s_{2}=\lambda_{2}\left(h_{l}, p\right),
$$

and the admissibility condition is satisfied if $p>p_{l}$, since $s_{2}(p)$ is non-increasing.

By easy computations, one can check that $p-1$ does not change sign across 1 -shocks, and similarly that the 2 -shocks do not cross the line $h=0$. These lines, $p=1$ and $h=0$, are contact discontinuity (shock/rarefaction) curves for the $1^{\text {st }}$ and $2^{\text {nd }}$ family, respectively.

In summary, the set of states that can be connected to $\left(h_{l}, p_{l}\right)$ by an entropy satisfying 1 -wave or 2-wave for the homogeneous system is given in Figure 2.
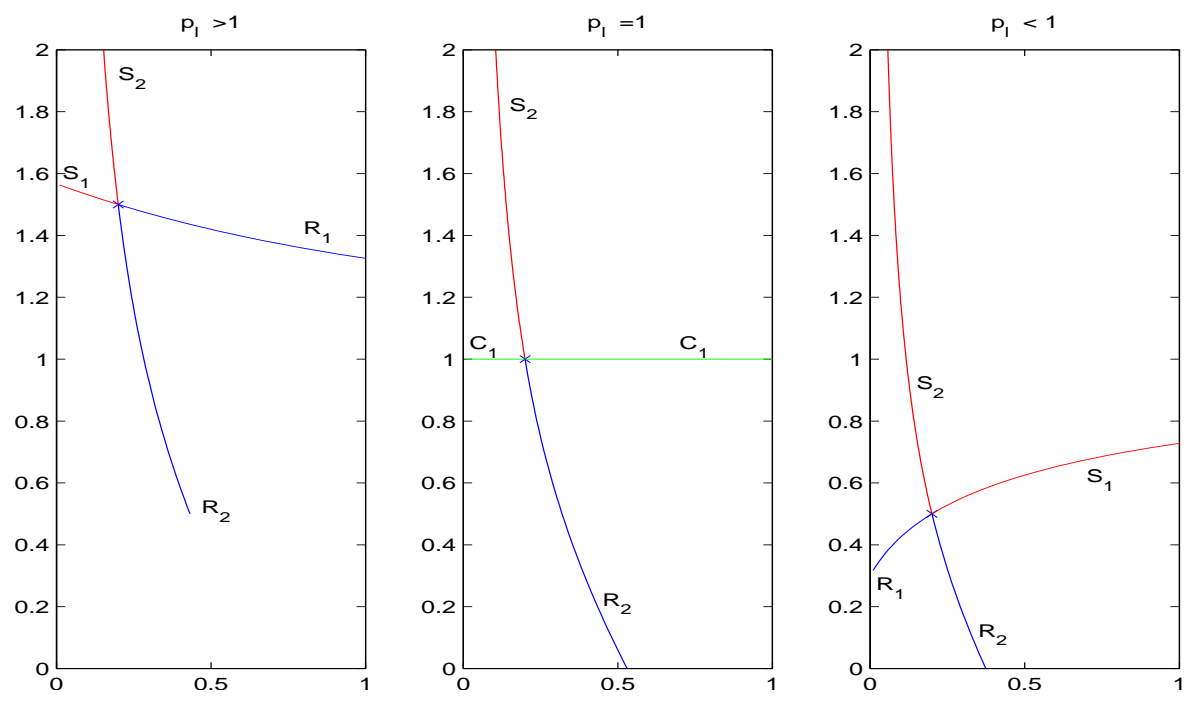

Figure 2: The set of states that can be connected to $\left(h_{l}, p_{l}\right)$ (marked by $*$ in the plots) by an entropy satisfying 1 -wave or 2 -wave for the homogeneous system. Here "R" stands for rarefaction wave, "S" for shock wave and "C" for contact discontinuity (i.e., shock/rarefaction wave).

Remark. We observe that the second characteristic family is genuinely nonlinear for $h>0$, but the first one is neither linearly degenerate, nor genuinely nonlinear in the domain $\Omega$. This situation may lead, in general, to the appearance of composite waves. However, it is crucial to observe that, in our case, composite waves never appear: any Riemann problem is solved by 
at most one simple wave of the first family and at most one simple wave of the second family. Hence, interaction of two waves generates at most two waves, one from each family.

Indeed, observe that the line $p=1$ separates the domain into two invariant regions from the point of view of Riemann problem, because the Hugoniot curve of the first family through a point $\left(h_{l}, p_{l}\right)$ where $p_{l} \neq 1$ will never cross that line. In each invariant region, since the system is genuinely nonlinear, there will be no composite waves.

In detail, let $\left(h_{l}, p_{l}\right)$ and $\left(h_{r}, p_{r}\right)$ be the left and right states of the Riemann problem, respectively. If $p_{l}>1$ and $p_{r}>1$, so will the solution of the Riemann problem, that is, $p>1$ for the intermediate state. Same is true for $p_{l}<1$ and $p_{r}<1$. Then, each 1 -wave will be a simple wave. If $p_{l}=1$ then the 1 -wave will be a contact discontinuity, with $p_{m}=1$. Finally, if $p_{l}$ and $p_{r}$ are on different sides of the line $p=1$, then the middle state $p_{m}$ will be on the same side as $p_{l}$, since the rarefaction curve or shock curve of the first family won't cross the line $p=1$, so the 1-wave will be again a simple wave. Since the second family is genuinely nonlinear, in all cases $\left(h_{m}, p_{m}\right)$ will be connected to $\left(h_{r}, p_{r}\right)$ by a simple 2 -wave.

\section{$3 \quad$ Time step estimates and interaction estimates}

Approximate solutions to the Cauchy problem (1.2), (1.3) are defined through a operator splitting method. Fix a time step $\Delta t \geq 0$ and consider the sequence of times $t_{k}=k \Delta t$. Let $\left(h^{\Delta}, p^{\Delta}\right)$ be an approximate solution, constructed as follows. On each subinterval $\left[t_{k-1}, t_{k}\right.$ [ the functions $\left(h^{\Delta}, p^{\Delta}\right)$ provide an approximate solution to the system of conservation laws

$$
\left\{\begin{aligned}
h_{t}-(h p)_{x} & =0 \\
p_{t}+((p-1) h)_{x} & =0
\end{aligned}\right.
$$

constructed by a wave-front tracking algorithm $[8,4,5]$. Moreover, in order to account for the source term, at each time $t_{k}$ the functions are redefined in the following way

$$
\left\{\begin{array}{l}
h^{\Delta}\left(t_{k}\right)=h^{\Delta}\left(t_{k}-\right)+\Delta t\left[p^{\Delta}\left(t_{k}-\right)-1\right] h^{\Delta}\left(t_{k}-\right), \\
p^{\Delta}\left(t_{k}\right)=p^{\Delta}\left(t_{k}-\right) .
\end{array}\right.
$$

In the remainder of this section we study how the strength of waves changes at a time $t_{k}$ where the source term is inserted, and at a time $\tau$ where two wave fronts interact. A key observation is that, in our particular system, the second characteristic field (called as $p$-field) is linearly degenerate along its integral curve $\{h=0\}$, and it is a straight line. The standard wave interaction estimates valid for $2 \times 2$ systems of conservation laws can thus be considerably improved, when $h \approx 0$.

Since the state space is two-dimensional, it is convenient to use a global system of Riemann coordinates:

Definition 1 (Riemann coordinates). Given a point $(h, p) \in \mathbb{R}_{+} \times \mathbb{R}_{+}$, let $(H, 0)$ be the point on the $h$-axis connected with $(h, p)$ by a 2 -characteristic curve, and let $(0, P)$ be the point on the $p$-axis connected to $(h, p)$ by a 1 -characteristic curve. Then the functions $(H, P)$ form a coordinate system of Riemann invariants of the point $(h, p)$. 
The change of variables $(h, p) \mapsto(H, P)$ satisfies the following

$$
\begin{array}{cc}
\frac{\partial H}{\partial h}>0, & \frac{\partial P}{\partial p}>0, \\
\frac{\partial H}{\partial p}=\frac{\lambda_{2}}{\lambda_{2}+1} \frac{\partial H}{\partial h}, & \frac{\partial P}{\partial h}=\frac{\lambda_{1}+1}{\lambda_{1}} \frac{\partial P}{\partial p},
\end{array}
$$

where the last two identities follow from $\nabla H \cdot r_{2}=0=\nabla P \cdot r_{1}$. The inverse transformation satisfies $\frac{\partial h}{\partial H}>0, \frac{\partial p}{\partial P}>0$.

Wave strengths are measured by the jumps between the corresponding Riemann coordinates of the left and right states, as follows. Given a wave front with left and right states $\left(h_{l}, p_{l}\right)$ and $\left(h_{r}, p_{r}\right)$, let $\left(H_{l}, P_{l}\right)$ and $\left(H_{r}, P_{r}\right)$ be the corresponding Riemann coordinates; then, if it is a wave of the $1^{\text {st }}$ family then its strength is measured by $\sigma_{h}=H_{r}-H_{l}$, and if it is a wave of the $2^{n d}$ family then its strength is measured by $\sigma_{p}=P_{r}-P_{l}$. Note that 2-rarefaction waves have negative strength, so 2-shock waves have positive strength (jump upward), and 1-rarefaction waves have positive strength for $p>1$ and negative strength for $p<1$.

Throughout the paper, the waves of the $1^{\text {st }}$ and $2^{n d}$ families are called $h$-waves and $p$-waves respectively. In the following estimates, we use $\mathcal{O}(1)$ to denote a quantity whose absolute value is bounded.

Lemma 1 (Time step estimates). Consider a wave front located at a point $x$, with $\left(h_{l}, p_{l}\right)$ and $\left(h_{r}, p_{r}\right)$ as the left and right state, respectively. After the time step (3.2) is accomplished, the Riemann problem determined by the jump at $x$ will be solved by two waves, say of strengths $\sigma_{h}^{+}, \sigma_{p}^{+}$.

If the initial jump is of the first family, with strength $\sigma_{h}$, we have

$$
\sigma_{h} \quad \Longrightarrow \quad\left\{\begin{array}{l}
\sigma_{h}^{+}=\sigma_{h}+\mathcal{O}(1) \cdot \Delta t \cdot\left|p_{l}-1\right| \cdot\left|\sigma_{h}\right|, \\
\sigma_{p}^{+}=\mathcal{O}(1) \cdot \Delta t \cdot\left|p_{l}-1\right| \cdot\left|\sigma_{h}\right| .
\end{array}\right.
$$

On the other hand, if the initial jump is of the second family, with strength $\sigma_{p}$, we have

$$
\sigma_{p} \quad \Longrightarrow \quad\left\{\begin{array}{l}
\sigma_{h}^{+}=\mathcal{O}(1) \cdot \Delta t \cdot h_{l}\left|\sigma_{p}\right|, \\
\sigma_{p}^{+}=\sigma_{p}+\mathcal{O}(1) \cdot \Delta t \cdot h_{l}\left|\sigma_{p}\right| .
\end{array}\right.
$$

Same estimates hold if one replaces $p_{l}$ with $p_{r}$ and $h_{l}$ with $h_{r}$ in (3.4) and (3.5), respectively.

Proof. Let $U_{l}=\left(h_{l}, p_{l}\right), U_{r}=\left(h_{r}, p_{r}\right)$ be the states on the left, right of the wave-front before the time step (3.2) and let $U_{l}^{+}, U_{r}^{+}$be the corresponding states after (3.2) is applied. To obtain (3.4), we define the mapping

$$
\Phi\left(\sigma_{h}, \Delta t, p_{l}\right)=\left(\sigma_{h}^{+}, \sigma_{p}^{+}\right)-\left(\sigma_{h}, 0\right) .
$$

If $p_{l}=1$, the $p_{r}=1$ and we trivially have $\Phi\left(\sigma_{h}, \Delta t, 1\right)=(0,0)$.

If $p_{l} \neq 1$ then $p_{r} \neq 1$, and we have the identities

$$
\Phi\left(0, \Delta t, p_{l}\right)=\Phi\left(\sigma_{h}, 0, p_{l}\right)=(0,0)
$$


therefore we get

$$
\begin{aligned}
\left|\Phi\left(\sigma_{h}, \Delta t, p_{l}\right)\right| & =\left|\int_{0}^{\Delta t} \partial_{\xi} \Phi\left(\sigma_{h}, \xi, p_{l}\right)-\partial_{\xi} \Phi\left(0, \xi, p_{l}\right) d \xi\right| \\
& =\left|\int_{0}^{\Delta t} \int_{0}^{\sigma_{h}} \partial_{\xi \eta}^{2} \Phi\left(\eta, \xi, p_{l}\right)-\partial_{\xi \eta}^{2} \Phi(\eta, \xi, 1) d \eta d \xi\right| \\
& \leq \mathcal{O}(1) \cdot\left|p_{l}-1\right|\left|\sigma_{h}\right| \Delta t,
\end{aligned}
$$

which implies (3.4).

The estimate (3.5) can be obtained in a similar way. Define the map

$$
\tilde{\Phi}\left(\sigma_{p}, \Delta t, h_{l}\right) \doteq\left(\sigma_{h}^{+}, \sigma_{p}^{+}\right)-\left(0, \sigma_{p}\right) .
$$

We see that if $h_{l}=0$, then $h_{r}=0$ so the source term vanishes on both sides of the wave. We have the identities

$$
\tilde{\Phi}\left(0, \Delta t, h_{l}\right)=\tilde{\Phi}\left(\sigma_{p}, 0, h_{l}\right)=\tilde{\Phi}\left(\sigma_{p}, \Delta t, 0\right)=(0,0) .
$$

By a same argument as for $\Phi$, we get (3.5).

The proof for the estimates with $p_{r}, h_{r}$ in (3.4), (3.5) is entirely similar; we omit the details.

Before we establish the wave interaction estimates, we need a technical lemma. The following lemma estimates how much the Rankine-Hugoniot condition fails to be satisfied along a $p$ characteristic curve, and how close the $p$-characteristic curve and Hugoniot locus are to each other as $h$ approaches 0 . This lemma is essential for the proof of the interaction estimates.

Lemma 2 Given $U_{o}=\left(h_{o}, p_{o}\right) \in \Omega$, let $U^{*}$ be a point on the $p$-characteristic curve through $U_{o}$. Then

$$
F\left(U^{*}\right)-F\left(U_{o}\right)-\bar{\lambda}_{2}\left(U^{*}-U_{o}\right)=\mathcal{O}(1) \cdot h_{o}^{2},
$$

where $F=(-h p,(p-1) h)^{t}$ is the flux function of the balance laws (1.2) and $\bar{\lambda}_{2}$ is the average of the $2^{\text {nd }}$ eigenvalue along the $p$-characteristic curve between $U_{o}$ and $U^{*}$.

Furthermore, there exists a point $U$ on the p-Hugoniot locus through $U_{o}$ such that

$$
\left|U-U^{*}\right|=\mathcal{O}(1) \cdot h_{o}^{2} .
$$

Proof. Observe that by construction we have, for some $a, b$

$$
\begin{aligned}
U^{*}-U_{o} & =\int_{a}^{b} r_{2}(U(\theta)) d \theta, \\
F\left(U^{*}\right)-F\left(U_{o}\right) & =\int_{a}^{b} \lambda_{2}(U(\theta)) \cdot r_{2}(U(\theta)) d \theta,
\end{aligned}
$$

where the integrals are computed along the $p$-characteristic curve through $U_{o}$. Consider the average eigenvalue $\bar{\lambda}_{2}$ and the average eigenvector $\bar{r}_{2}$ along the $p$-characteristic curve, defined as

$$
\begin{aligned}
& \bar{\lambda}_{2} \doteq \frac{1}{b-a} \int_{a}^{b} \lambda_{2}(U(\theta)) d \theta \\
& \bar{r}_{2} \doteq \frac{1}{b-a} \int_{a}^{b} r_{2}(U(\theta)) d \theta=\frac{1}{b-a}\left(U^{*}-U_{o}\right),
\end{aligned}
$$


we have

$$
F\left(U^{*}\right)-F\left(U_{o}\right)-\bar{\lambda}_{2}\left(U^{*}-U_{o}\right)=\int_{a}^{b}\left(\lambda_{2}(U(\theta))-\bar{\lambda}_{2}\right) \cdot r_{2}(U(\theta)) d \theta .
$$

Since the average of $\left(\lambda_{2}(U(\theta))-\bar{\lambda}_{2}\right)$ over $(a, b)$ along the $p$-characteristic curve is zero, we have

$$
F\left(U^{*}\right)-F\left(U_{o}\right)-\bar{\lambda}_{2}\left(U^{*}-U_{o}\right)=\int_{a}^{b}\left(\lambda_{2}(U(\theta))-\bar{\lambda}_{2}\right) \cdot\left(r_{2}(U(\theta))-\bar{r}_{2}\right) d \theta
$$

To get an estimate on the integrand in (3.8), we observe that

$$
\lambda_{2}(U(\theta))-\bar{\lambda}_{2}=\mathcal{O}(1) \cdot h_{o} .
$$

This is because that the $p$-field is linearly degenerate at $h_{o}=0$, so $\lambda_{2}$ approaches a constant (as a consequence $\lambda_{2}(U(\theta))-\bar{\lambda}_{2}$ vanishes) as $h_{o} \rightarrow 0$, and also $\lambda_{2}$ depends smoothly on $h_{o}$. Furthermore, we also have

$$
r_{2}(U(\theta))-\bar{r}_{2}=\mathcal{O}(1) \cdot h_{o}
$$

since the 2-characteristic curve is a straight line at $h_{o}=0$ and it depends continuously on $h_{o}$. Then, using (3.9) and (3.10) in (3.8), we get (3.6).

To establish (3.7), we observe that it follows from the global existence of the Hugoniot curves of the first family. In detail, we consider two cases. First, let $p$ and $p_{o}$ be sufficiently far away from each other. We need to show that the Hugoniot loci are globally well-defined. Indeed, given $\left(h_{o}, p_{o}\right)$ and $p$, one can solve the Rankine-Hugoniot equations for $h$ and shock speed $s$,

$$
\begin{aligned}
-h p+h_{o} p_{o}-s\left(h-h_{o}\right) & =0, \\
(p-1) h-\left(p_{o}-1\right) h_{o}-s\left(p-p_{o}\right) & =0 .
\end{aligned}
$$

The Jacobian matrix (considering $h$ and $s$ as the unknowns) of this transformation is

$$
\left(\begin{array}{ll}
-p-s & -\left(h-h_{o}\right) \\
p-1 & -\left(p-p_{o}\right)
\end{array}\right)
$$

whose determinant is uniformly non zero since both $h-h_{o}$ and $s(\approx h)$ are arbitrary small.

Secondly, when $p$ and $p_{o}$ are very close to each other, it follows from Glimm-Lax type argument [19]. Indeed, let $\bar{A}\left(U^{*}, U_{o}\right)$ be the average Jacobian of $F$ from $U_{o}$ to $U^{*}$ along the $p$ characteristic curve, and let $\bar{l}_{i}, \bar{r}_{i}, \bar{\lambda}_{i}$ be the normalized left and right eigenvectors and eigenvalues of $\bar{A}\left(U^{*}, U_{o}\right)$, respectively. We can rewrite (3.6) as

$$
\left(\bar{A}\left(U^{*}, U_{o}\right)-\bar{\lambda}_{2} I\right)\left(U^{*}-U_{o}\right)=\mathcal{O}(1) \cdot h_{o}^{2} .
$$

Taking scalar product with $\bar{l}_{1}\left(U^{*}, U_{o}\right)$ on the left, we get

$$
\left(\bar{\lambda}_{1}\left(U^{*}, U_{o}\right)-\bar{\lambda}_{2}\right)<\bar{l}_{1}\left(U^{*}, U_{o}\right), U^{*}-U_{o}>=\mathcal{O}(1) \cdot h_{o}^{2} .
$$

First we see that $\lambda_{2}\left(U^{*}, U_{o}\right) \geq 0$; furthermore, since $p^{*}$ and $p_{o}$ are positive, then $\bar{\lambda}_{1}\left(U^{*}, U_{o}\right)<0$. Therefore we have

$$
\left|\bar{\lambda}_{1}\left(U^{*}, U_{o}\right)-\bar{\lambda}_{2}\left(U^{*}, U_{o}\right)\right|>0
$$

and this inequality holds uniformly on domains where $p$ is bounded away from zero. Therefore (3.11) implies

$$
\mathcal{L}\left(U^{*}\right) \doteq\left\langle\bar{l}_{1}\left(U^{*}, U_{o}\right), U^{*}-U_{o}\right\rangle=\mathcal{O}(1) \cdot h_{o}^{2}
$$


Observe that for small $U^{*}-U_{o}$ we have

$$
\nabla \mathcal{L}\left(U^{*}\right)=\nabla_{U} \bar{l}_{1}\left(U^{*}, U_{o}\right)^{t}\left(U^{*}-U_{o}\right)+\bar{l}_{1}\left(U^{*}, U_{o}\right) \neq(0,0),
$$

because $\bar{l}_{1}$ is non-zero. By implicit function theorem, for sufficiently small $h_{o}$, there exists a $U$ near $U^{*}$, with

$$
\left|U-U^{*}\right|=\mathcal{O}(1) \cdot h_{o}^{2}
$$

such that

$$
\left\langle\bar{l}_{1}\left(U, U_{0}\right), U-U_{o}\right\rangle=0 .
$$

This means $U-U_{o}$ is a 2-eigenvector of $\bar{A}\left(U, U_{o}\right)$, so $U$ lies on the 2-Hugoniot locus through $U_{o}$, completing the proof.

In next lemma we establish the wave interaction estimates for our model, which improved the standard ones.

Lemma 3 (Interaction Estimates). Consider two interacting wave fronts, with left, middle, and right states $\left(h_{l}, p_{l}\right),\left(h_{m}, p_{m}\right),\left(h_{r}, p_{r}\right)$ before interaction, respectively, and $h_{\max }=$ $\max \left\{h_{l}, h_{m}, h_{r}\right\}$.

(i) Assume that two p-waves of strength $\sigma_{p}$ and $\tilde{\sigma}_{p}$ interact. Then the strengths $\sigma_{h}^{+}$and $\sigma_{p}^{+}$of the outgoing waves satisfy

$$
\left|\sigma_{h}^{+}\right|+\left|\sigma_{p}^{+}-\left(\sigma_{p}+\tilde{\sigma}_{p}\right)\right|=\mathcal{O}(1) \cdot h_{l} \cdot\left|\sigma_{p} \tilde{\sigma}_{p}\right| .
$$

(ii) If two $h$-waves of size $\sigma_{h}$ and $\tilde{\sigma}_{h}$ interact, then the strengths $\sigma_{h}^{+}$and $\sigma_{p}^{+}$of the outgoing waves satisfy

$$
\left|\sigma_{h}^{+}-\left(\sigma_{h}+\tilde{\sigma}_{h}\right)\right|+\left|\sigma_{p}^{+}\right|=\mathcal{O}(1) \cdot\left|p_{l}-1\right|\left(\left|\sigma_{h}\right|+\left|\tilde{\sigma}_{h}\right|\right) \cdot\left|\sigma_{h} \tilde{\sigma}_{h}\right|,
$$

or equivalently

$$
\left|\sigma_{h}^{+}-\left(\sigma_{h}+\tilde{\sigma}_{h}\right)\right|+\left|\sigma_{p}^{+}\right|=\mathcal{O}(1) \cdot \min \left\{\left|p_{l}-1\right|,\left|p_{m}-1\right|\right\} \cdot\left(\left|\sigma_{h}\right|+\left|\tilde{\sigma}_{h}\right|\right) \cdot\left|\sigma_{h} \tilde{\sigma}_{h}\right| \cdot
$$

(iii) If two waves of different families interact, say with strengths $\sigma_{h}, \sigma_{p}$, then the strengths $\sigma_{h}^{+}$ and $\sigma_{p}^{+}$of the waves emerging from the interaction satisfy

$$
\left|\sigma_{h}^{+}-\sigma_{h}\right|+\left|\sigma_{p}^{+}-\sigma_{p}\right|=\mathcal{O}(1) \cdot h_{\max } \cdot\left|\sigma_{h} \sigma_{p}\right| .
$$

Proof. (i) First we observe that, if $h_{l}=0$, the also $h_{m}=h_{r}=0$ since $h=0$ is a $p$ shock/rarefaction curve. Then $\sigma_{p}^{+}=\sigma_{p}+\tilde{\sigma}_{p}$ and $\sigma_{h}^{+}=0$, and (3.14) is trivially satisfied. In general, we define the mapping

$$
\Phi\left(h_{l}, \sigma_{p}, \tilde{\sigma}_{p}\right) \doteq\left(\sigma_{h}^{+}, \sigma_{p}^{+}-\sigma_{p}-\tilde{\sigma}_{p}\right) .
$$

We remark that, throughout the proof, $\Phi$ will be used to denote different mappings of similar type, without causing confusion.

Notice that $\Phi$ is smooth w.r.t. $h_{l}$ and twice continuously differentiable w.r.t. $\sigma_{h}, \tilde{\sigma}_{h}$. The following identities hold:

$$
\Phi\left(0, \sigma_{p}, \tilde{\sigma}_{p}\right)=\Phi\left(h_{l}, \sigma_{p}, 0\right)=\Phi\left(h_{l}, 0, \tilde{\sigma}_{p}\right)=(0,0)
$$


therefore we have

$$
\Phi\left(h_{l}, \sigma_{p}, \tilde{\sigma}_{p}\right)=\int_{0}^{\sigma_{p}} \int_{0}^{\tilde{\sigma}_{p}} \frac{\partial^{2} \Phi}{\partial \sigma_{p} \partial \tilde{\sigma}_{p}}\left(h_{l}, r, s\right) d r d s
$$

where the integrand vanishes if $h_{l}=0$ and is Lipschitz continuous w.r.t. $h_{l}$. Hence we get (3.14).

(ii) We observe that, if $p_{l}=p_{m}=p_{r}=1$, then $\sigma_{h}^{+}=\sigma_{h}+\tilde{\sigma}_{h}$ and $\sigma_{p}^{+}=0$, and the estimate (3.15) is trivial. In the general case, the three quantities $p_{l}, p_{m}, p_{r}$ are either all $>1$, or all $<1$. To fix the ideas, we shall thus assume $p_{l}, p_{m}, p_{r}>1$, the other case being similar. Introduce the mapping

$$
\Phi\left(h_{l}, p_{l}, \sigma_{h}, \tilde{\sigma}_{h}\right) \doteq\left(\sigma_{h}^{+}-\sigma_{h}-\tilde{\sigma}_{h}, \sigma_{p}^{+}\right) .
$$

Notice that $\Phi$ is smooth w.r.t. $h_{l}, p_{l}$, and twice continuously differentiable w.r.t. $\sigma_{h}, \tilde{\sigma}_{h}$. Moreover, $\Phi$ is separately smooth restricted to each of the four quadrants

$$
\begin{array}{ll}
D_{1}=\left\{\sigma_{h} \geq 0, \tilde{\sigma}_{h} \geq 0\right\}, & D_{2}=\left\{\sigma_{h} \leq 0, \tilde{\sigma}_{h} \geq 0\right\}, \\
D_{3}=\left\{\sigma_{h} \leq 0, \tilde{\sigma}_{h} \leq 0\right\}, & D_{4}=\left\{\sigma_{h} \geq 0, \tilde{\sigma}_{h} \leq 0\right\} .
\end{array}
$$

Since $\Phi\left(h_{l}, p_{l}, \sigma_{h}, \tilde{\sigma}_{h}\right)=(0,0)$ on $D_{1}$, we have

$$
\frac{\partial^{2} \Phi}{\partial \sigma_{h} \partial \tilde{\sigma}_{h}}\left(h_{l}, p_{l}, 0,0\right)=(0,0) .
$$

Using the previous identity and the following ones:

$$
\Phi\left(h_{l}, 1, \sigma_{h}, \tilde{\sigma}_{h}\right)=\Phi\left(h_{l}, p_{l}, 0, \tilde{\sigma}_{h}\right)=\Phi\left(h_{l}, p_{l}, \sigma_{h}, 0\right)=(0,0),
$$

we can write

$$
\begin{aligned}
\Phi\left(h_{l}, p_{l}, \sigma_{h}, \tilde{\sigma}_{h}\right) & =\int_{0}^{\sigma_{h}} \int_{0}^{\tilde{\sigma}_{h}} \frac{\partial^{2} \Phi}{\partial \sigma_{h} \partial \tilde{\sigma}_{h}}\left(h_{l}, p_{l}, r, \tilde{r}\right) d r d \tilde{r} \\
& =\int_{0}^{\sigma_{h}} \int_{0}^{\tilde{\sigma}_{h}}\left[\int_{1}^{p_{l}} \int_{0}^{1} \frac{\partial}{\partial p_{l}}\left[r \frac{\partial}{\partial \sigma_{h}}+\tilde{r} \frac{\partial}{\partial \tilde{\sigma}_{h}}\right] \frac{\partial^{2} \Phi}{\partial \sigma_{h} \partial \tilde{\sigma}_{h}}\left(h_{l}, p, \theta r, \theta \tilde{r}\right) d \theta d p\right] d r d \tilde{r} .
\end{aligned}
$$

Observing that in the above integrand one always has $|r|+|\tilde{r}| \leq\left|\sigma_{h}\right|+\left|\tilde{\sigma}_{h}\right|$, we obtain (3.15).

Finally, note that with the same type of arguments one obtains the same estimate as (3.15) with $p_{m}$ instead of $p_{l}$. Therefore (3.16) follows.

(iii) We first recall that, by using Riemann coordinates, one has the standard cubic estimate

$$
\left|\sigma_{h}^{+}-\sigma_{h}\right|+\left|\sigma_{p}^{+}-\sigma_{p}\right|=\mathcal{O}(1) \cdot\left|\sigma_{h} \sigma_{p}\right|\left(\left|\sigma_{p}\right|+\left|\sigma_{h}\right|\right)
$$

However, this is useless for our problem, because though $\left|\sigma_{h}\right|=\mathcal{O}(1) \cdot\|h\|_{\infty}$ is small, the strength of the $p$-wave can be large. Having a cubic rather than a quadratic estimate thus would not help. The proof for the estimate in (3.17) heavily relies on Lemma 2, i.e., the $p$-characteristic and Hugoniot curves are very close to each other for small $h$.

We introduce the mapping

$$
\Phi\left(h_{l}, p_{l}, \sigma_{h}, \sigma_{p}\right) \doteq\left(\sigma_{h}^{+}, \sigma_{p}^{+}\right)-\left(\sigma_{h}, \sigma_{p}\right) .
$$


Observe that $\Phi$ is smooth w.r.t. $h_{l}, p_{l}$, and twice continuously differentiable w.r.t. $\sigma_{h}, \sigma_{p}$, with Lipschitz-continuous second derivatives. Moreover, the following identities hold trivially

$$
\Phi\left(h_{l}, p_{l}, \sigma_{h}, 0\right)=\Phi\left(h_{l}, p_{l}, 0, \sigma_{p}\right)=(0,0),
$$

hence

$$
\Phi\left(h_{l}, p_{l}, \sigma_{h}, \sigma_{p}\right)=\int_{0}^{\sigma_{p}} \int_{0}^{\sigma_{h}} \frac{\partial^{2} \Phi}{\partial \sigma_{p} \partial \sigma_{h}}\left(h_{l}, p_{l}, r, s\right) d r d s
$$

which leads to the standard cubic estimate (3.18), thank also to

$$
\frac{\partial^{2} \Phi}{\partial \sigma_{p} \partial \sigma_{h}}\left(h_{l}, p_{l}, 0,0\right)=(0,0) \text {. }
$$

Now we claim that

$$
\frac{\partial^{2} \Phi}{\partial \sigma_{p} \partial \sigma_{h}}\left(0, p_{l}, 0, \sigma_{p}\right)=(0,0)
$$

If (3.20) holds, then by (3.19) and the Lipschitz continuity of the second derivatives we get

$$
\left|\Phi\left(h_{l}, p_{l}, \sigma_{h}, \sigma_{p}\right)\right| \leq \mathcal{O}(1) \int_{0}^{\left|\sigma_{p}\right|} \int_{0}^{\left|\sigma_{h}\right|}\left(h_{l}+r\right) d r d s=\mathcal{O}(1)\left(h_{l}+\left|\sigma_{h}\right|\right)\left|\sigma_{h} \sigma_{p}\right|,
$$

which implies (3.17) since $h_{l}+\left|\sigma_{h}\right|=\mathcal{O}(1) h_{\max }$.

To establish (3.20), we consider two cases, depending on the type of $p$-waves.

a. Consider $\sigma_{p}<0$, i.e., the $p$-wave is a rarefaction. Due to the genuine nonlinearity of the field, $p$-waves do not change type after interaction, so $\sigma_{p}^{+}<0$ is also a rarefaction. We trivially have

$$
\sigma_{h}^{+}-\sigma_{h}=0 .
$$

We still need an estimate on the term $\sigma_{p}^{+}-\sigma_{p}$, depends on the types of $h$-waves. If they are all rarefaction waves, then it's trivial since $\sigma_{p}^{+}-\sigma_{p}=0$. If one of them is a shock, then by the fact that the $h$-shock and rarefaction curves through the same point share the $2^{\text {nd }}$ order tangency at the point, and by the continuity of the derivatives, we achieve the desired estimate

$$
\sigma_{p}^{+}-\sigma_{p}=\mathcal{O}(1) \cdot\left|\sigma_{p}\right| \cdot\left|\sigma_{h}\right|^{2}
$$

b. It remains to consider the case where the $p$-waves $\sigma_{p}>0$ and $\sigma_{p}^{+}>0$ are shocks. In order to show (3.20), we see that it suffices to show

$$
\frac{\partial \Phi}{\partial \sigma_{h}}\left(0, p_{l}, 0, \sigma_{p}\right)=(0,0)
$$

Thanks to Lemma 2, the distance between a $p$-characteristic curve and Hugoniot curve is of order $\mathcal{O}(1) \cdot \sigma_{h}^{2}$, which implies $(3.22)$, completing the proof. 
We conclude this section with a remark about the change of $P$ along Hugoniot curves of the first family.

Recalling Definition 1, let $(H, P)$ be the Riemann coordinates of a point on the Hugoniot curve of the first family originated from $\left(H_{l}, P_{l}\right)$, and let $\sigma=H-H_{l}$ be the size of the wave. Then one has the following estimate:

$$
P-P_{l}=\mathcal{O}(1) \cdot\left|P_{l}-1\right| \cdot \sigma^{2} .
$$

Here the term $\sigma^{2}$ comes from the second order tangency of the shock and rarefaction curves; the term $\left|P_{l}-1\right|$ is justified by observing that the function $\Phi\left(H_{l}, P_{l} ; \sigma\right) \doteq P-P_{l}$ vanishes identically if $P_{l}=1$.

Moreover, for small $\sigma$, one has the following estimate:

$$
P-P_{l}=\mathcal{O}(1) \cdot \min \left\{\left|P_{l}-1\right|,|P-1|\right\} \cdot \sigma^{2} .
$$

Indeed, by (3.23) one has

$$
P-1=\left(P-P_{l}\right)+\left(P_{l}-1\right)=\left(P_{l}-1\right)\left(1+\mathcal{O}(1) \sigma^{2}\right) .
$$

\section{A priori estimates}

The goal of the present paper is the global existence of large BV solutions, for all times $t \geq 0$. This will be established by deriving the following global a priori bounds for the approximate solutions:

- the norms $\|h(t, \cdot)\|_{\mathbf{L}^{1}}$ and $\|p(t, \cdot)-1\|_{\mathbf{L}^{1}}$;

- the lower bound on $p$, i.e., the quantity $\inf _{x} p(t, x)$;

- the uniform bounds on $h$ and $p$, i.e., the quantities $\|h(t, \cdot)\|_{\mathbf{L}^{\infty}}$ and $\|p(t, \cdot)\|_{\mathbf{L}^{\infty}}$;

- the total variations Tot.Var. $\{h(t, \cdot)\}$ and Tot.Var. $\{p(t, \cdot)\}$.

Approximate solutions will be obtained by constructing a front tracking approximation to the conservation laws (3.1) on each time interval $\left[t_{k-1}, t_{k}\right)$, as in $[8$, Chapter 7$]$ or $[9,4]$ for the $2 \times 2$ version, and then updating the solution at time $t=t_{k}$ according to (3.2).

In the remainder of this section, we will give the main ideas of establishing these a priori estimates. All estimates will be made rigorous later in Section 5 where we complete the proof of the global existence of large BV solutions.

\subsection{The $\mathbf{L}^{1}$ bound on $p(t, \cdot)-1$ and $h(t, \cdot)$.}

We establish this a priori estimate on the weak solutions of (1.2). In terms of the new variable $q=p-1$, the system (1.2) takes the form

$$
\left\{\begin{aligned}
h_{t}-((q+1) h)_{x} & =q h, \\
q_{t}+(h q)_{x} & =0,
\end{aligned}\right.
$$


with initial data $(\bar{h}, \bar{q})$ that satisfies $\bar{h} \geq 0$ and $\bar{q}>-1$. According to the second equation in (4.1), the quantity $q \doteq p-1$ satisfies a transport equation, with speed $h$. Therefore, its $\mathbf{L}^{1}$ norm is non-increasing in time; we thus have

$$
\|q(t, \cdot)\|_{\mathbf{L}^{1}} \leq\|\bar{q}\|_{\mathbf{L}^{1}} \quad \Rightarrow \quad\|p(t, \cdot)-1\|_{\mathbf{L}^{1}} \leq\|\bar{p}-1\|_{\mathbf{L}^{1}} \quad \text { for all } t \geq 0 .
$$

Actually, the $\mathbf{L}^{1}$ norm of $p-1$ can decrease in the presence of a shock with left and right states of $q$ of opposite signs. From Figure 2 we see this can only happen with a shock in the $2^{\text {nd }}$ family, satisfying $p_{l}<1, p_{r}>1$.

From the first equation in (4.1) we obtain

$$
\|h(t)\|_{\mathbf{L}^{1}} \leq \int_{0}^{t} \int(p(\tau, x)-1) h(\tau, x) d x d \tau=\int_{0}^{t} \int q(\tau, x) h(\tau, x) d x d \tau .
$$

Note that when $p<1$, the moving layer will deposit and get thinner, so $\|h(t)\|_{\mathbf{L}^{1}}$ will decrease. It will however increase where $p>1$. To control the increment, we consider the weighted functional

$$
\mathcal{I}^{h}(t) \doteq \int W(t, x) h(t, x) d x
$$

where the weight is defined as

$$
W(t, x) \doteq \exp \left\{\int_{-\infty}^{x}|p(t, y)-1| d y\right\}=\exp \left\{\int_{-\infty}^{x}|q(t, y)| d y\right\} \geq 1 .
$$

Note that the weight $W$ is uniformly bounded thanks to the bound on $\|q(t, \cdot)\|_{\mathbf{L}^{1}}$. Here the weight $W$ accounts for the erosion/deposition that the mass $h$ at $x$ may encounter in the future. From the bound on the $\mathbf{L}^{1}$ norm of $p-1$ in (4.2), we see that $W$ is positive and uniformly bounded for all time. Intuitively, after $h$ crosses some region with $p \neq 1$, the weighted functional $\mathcal{I}^{h}$ will decrease.

Indeed, observing that

$$
\frac{d}{d t} \int_{-\infty}^{x}|p(t, y)-1| d y \leq-|p(t, x)-1| h(t, x),
$$

we see that the product $W h$ satisfies the following inequality

$$
(W h)_{t}-(W h(q+1))_{x} \leq W h(q-|q|(h+q+1)) .
$$

Note the right hand side is $\leq 0$ for $h \geq 0$ and $q \geq-1$. Then, the integral of $W h$ is non-increasing in time and we immediately have

$$
\frac{d}{d t} \mathcal{I}^{h}(t) \leq \int_{-\infty}^{\infty} W h(q-|q|(h+q+1)) d x \leq 0
$$

From (4.7), we obtain the a priori bound

$$
\|h(t, \cdot)\|_{\mathbf{L}^{1}} \leq \mathcal{I}^{h}(t) \leq \mathcal{I}^{h}(0) \leq \exp \left(\|\bar{q}\|_{\mathbf{L}^{1}}\right) \cdot\|\bar{h}\|_{\mathbf{L}^{1}} .
$$




\subsection{The lower bound on $p$}

The bounds on the total variation, as well as the lower bound on $p$ and the pointwise bounds on $h, p$, rely on the following key observation: If all wave strengths are measured in terms of Riemann coordinates, then all the interaction estimates (3.14)-(3.17) contain the additional factor $\|h\|_{\mathbf{L}^{\infty}}$. Therefore, if the norm $\|h\|_{\mathbf{L}^{\infty}}$ remains sufficiently small, we can assume that the total strength of all new waves produced by interactions is as small as we like. In essence, the change in the total variation and in the $\mathbf{L}^{\infty}$ norms of $h, p$ is thus determined only by the source term in the first equation (4.1).

We now show how to achieve these a priori bounds, based on the estimates (3.4)-(3.5).

Let's first establish the lower bound on $p$. Let $H(h, p), P(h, p)$ be the Riemann coordinates of a point $(h, p)$ as in Definition 1. We claim that, neglecting small terms due to wave interactions, the quantity

$$
P_{\text {inf }}(t) \doteq \text { ess- } \inf _{x} P(t, x)
$$

is non-decreasing in time.

This a priori estimate is derived for smooth solutions of (1.2). Consider a 2-characteristic, say $t \mapsto x_{2}(t)$, with $\dot{x}_{2}(t)=\lambda_{2}(t) \geq 0$. Then one has

$$
\frac{d}{d t} P\left(t, x_{2}(t)\right)=\frac{\partial P}{\partial h} \cdot(p-1) h \geq 0 .
$$

Indeed, the geometry of the wave curves implies $\partial P / \partial h<0$ when $p<1$ and that $\partial P / \partial h>0$ when $p>1$. This shows that the quantity $P_{\text {inf }}$ is non-decreasing in time if the solution is smooth.

\subsection{The $\mathbf{L}^{\infty}$ bounds on $h$ and $p$.}

Toward the uniform bounds on $h$ and $p$, we consider the weights

$$
\begin{aligned}
V^{h}(t, x) & \doteq \exp \left(\kappa_{1} \cdot \int_{-\infty}^{x}|p(t, y)-1| d y\right) \\
V^{p}(t, x) & \doteq \exp \left(\kappa_{2} \cdot \int_{x}^{\infty} W(t, y) h(t, y) d y\right)
\end{aligned}
$$

for some suitable constants $\kappa_{1}$ and $\kappa_{2}$. Recall that $W$ is defined in (4.4). Thanks to the a priori bounds on the $\mathbf{L}^{1}$ norm of $h$ and $p-1$, we see that these two weights are well defined and bounded for all time. Furthermore, along a given smooth curve $x(t)$, by (4.5) we get

$$
\frac{d}{d t} V^{h}(t, x(t)) \leq-\kappa_{1} V^{h} \cdot|p-1| \cdot(h-\dot{x})
$$

while from (4.6) we have

$$
\frac{d}{d t} \int_{x}^{\infty} W(t, y) h(t, y) d y \leq-W(t, x) p(t, x) h(t, x)
$$

and therefore we obtain

$$
\frac{d}{d t} V^{p}(t, x(t)) \leq-\kappa_{2} V^{p} \cdot W h \cdot(\dot{x}+p)
$$


Again, let $H(h, p), P(h, p)$ be the Riemann coordinates of a point $(h, p)$ as in Definition 1 . We claim that, neglecting small terms due to wave interactions, the quantities

$$
\begin{aligned}
\mathcal{J}^{h}(t) & \doteq \operatorname{ess}-\sup _{x} V^{h}(t, x) H(t, x), \\
\mathcal{J}^{p}(t) & \doteq \operatorname{ess-} \sup _{x} V^{p}(t, x) P(t, x)
\end{aligned}
$$

are non-increasing in time.

First we will establish the bound on the quantity $\mathcal{J}^{p}(t)$, for smooth solutions of (1.2). Let's consider a 2-characteristic $t \mapsto x_{2}(t)$. Observe that the mass of $h$ is transported to the left with speed $-p$, while $\dot{x}_{2}=\lambda_{2} \geq 0$, so the relative speed of the mass of $h$ cross the 2-characteristic is $p+\lambda_{2}$. By (4.14), (4.4) and using the apriori bound on $p, P$ from below (let us denote them by $p_{\text {inf }}, P_{\text {inf }}$ respectively, both positive), we get

$$
\begin{aligned}
\frac{d}{d t}\left(V^{p}\left(t, x_{2}(t)\right) P\left(t, x_{2}(t)\right)\right) & =\left(\frac{d}{d t} V^{p}\left(t, x_{2}(t)\right)\right) \cdot P+V^{p} \cdot \frac{d}{d t}\left(P\left(t, x_{2}(t)\right)\right. \\
& \leq-\kappa_{2} V^{p} \cdot\left(p+\lambda_{2}\right) \cdot W h \cdot P+V^{p} \cdot \frac{\partial P}{\partial h}(p-1) h \\
& \leq V^{p} h\left(-\kappa_{2} p_{\text {inf }} P_{\text {inf }}+\frac{\partial P}{\partial h}(p-1)\right) \leq 0
\end{aligned}
$$

where the last inequality holds provided that the constant $\kappa_{2}$ is chosen large enough. This shows that the quantity $\mathcal{J}^{p}(t)$ is non-increasing.

Second, we derive the bound on the quantity $\mathcal{J}^{h}(t)$, also for the smooth solutions of (1.2). Let's consider a 1-characteristic $t \mapsto x_{1}(t)$, with $\dot{x}_{1}=\lambda_{1}$. We observe that the mass of $q=p-1$ is transported forward with speed $h$, while, by $(2.5), \dot{x}_{1}=\lambda_{1} \leq-p+h$, so the relative speed of the mass $q$ passing the 1-characteristic is bigger than $h-(-p+h)=p$. Hence, by (4.13) we have

$$
\begin{aligned}
\frac{d}{d t}\left(V^{h}\left(t, x_{1}(t)\right) H\left(t, x_{1}(t)\right)\right) & =\left(\frac{d}{d t} V^{h}\left(t, x_{1}(t)\right)\right) \cdot H+V^{h} \cdot \frac{d}{d t}\left(H\left(t, x_{1}(t)\right)\right. \\
& \leq-\kappa_{1} V^{h} \cdot p \cdot|p-1| H+V^{h} \cdot \frac{\partial H}{\partial h}(p-1) h \\
& \leq V^{h}|p-1| H\left(-\kappa_{1} p_{\text {inf }}+\mathcal{O}(1) \frac{\partial H}{\partial h}\right) \leq 0
\end{aligned}
$$

where the last inequality holds provided that the constant $\kappa_{1}$ is chosen large enough. This shows that the quantity $\mathcal{J}^{h}(t)$ is also non-increasing.

Since the weights $V^{h}$ and $V^{p}$ are uniformly bounded, we conclude that $\|H(t, \cdot)\|_{\mathbf{L}^{\infty}}$ and $\|P(t, \cdot)\|_{\mathbf{L}^{\infty}}$ are bounded for all time $t>0$, and so are $\|h(t, \cdot)\|_{\mathbf{L}^{\infty}}$ and $\|p(t, \cdot)\|_{\mathbf{L}^{\infty}}$.

\subsection{Wave front interactions: total wave strength and interaction potential}

We now study the effect of wave-front interactions, and derive a-priori bounds on the total variation. To clarify the main ideas, it is convenient first to consider the case without sources, as in (3.1), with initial data (1.3). If (1.4), (1.6) and (1.7) hold for some $\delta>0$ sufficiently small, we claim that the entropy-weak solution exists globally and satisfies a uniform BV bound. 
As usual, BV bounds are obtained by introducing a suitable wave strenght and wave interaction potential and checking that the sum (or some linear combination of them) is non-increasing along any solution of (3.1).

Let $u=(h, p): \mathbb{R} \mapsto \mathbb{R}^{2}$ be a piecewise constant mapping, with $h \geq 0$, and whose jumps consist of admissible shocks or rarefactions, for the system (3.1). Assume that at $x_{\alpha}$ the profile $u(\cdot)$ has a jump in the family $i_{\alpha} \in\{1,2\}$, with strength $\left|\sigma_{\alpha}\right|$ measured by the change in the corresponding Riemann coordinates. We then define the total strength of waves $V$ and the wave interaction potential $\mathcal{Q}$ by setting

$$
V=\sum_{\alpha}\left|\sigma_{\alpha}\right|, \quad \mathcal{Q}(u)=\mathcal{Q}_{h h}+\mathcal{Q}_{p p}+\mathcal{Q}_{p h}
$$

Here

$$
\mathcal{Q}_{h h}=\sum_{i_{\alpha}=i_{\beta}=1, x_{\alpha}<x_{\beta}} w_{\alpha, \beta}\left|\sigma_{\alpha}\right|\left|\sigma_{\beta}\right|,
$$

is the interaction potential of waves of the first family (i.e., $h$-waves). Since this first characteristic field is not genuinely nonlinear along the line $\{p=1\}$, we insert here the factor $w_{\alpha, \beta}$ defined as follows. If $\sigma_{\alpha}$ and $\sigma_{\beta}$ are two shocks, on the same side of the line $p=1$, then we set

$$
w_{\alpha, \beta}=\delta_{0} \cdot \min \left\{\left|P_{l}^{\alpha}-1\right|,\left|P_{l}^{\beta}-1\right|\right\}
$$

where $P_{l}^{\alpha}=P\left(x_{\alpha}-\right), P_{l}^{\beta}=P\left(x_{\beta}-\right)$ are the left limits of $P$ (Riemann coordinate) at $x_{\alpha}, x_{\beta}$ respectively, and $\delta_{0}>0$ is a small constant to be determined. In all other cases, we set $w_{\alpha, \beta}=0$. The other parts of the interaction potential are defined as usual:

$$
\mathcal{Q}_{p p}=\sum_{(\alpha, \beta) \in \mathcal{A}_{2}}\left|\sigma_{\alpha}\right|\left|\sigma_{\beta}\right|
$$

is the interaction potential of waves of the second family. Here $\mathcal{A}_{2}$ denotes the set of couples of waves of the second family, with $x_{\alpha}<x_{\beta}$, at least one of which is a shock. Finally,

$$
\mathcal{Q}_{p h}=\sum_{i_{\alpha}=2, i_{\beta}=1, x_{\alpha}<x_{\beta}}\left|\sigma_{\alpha}\right|\left|\sigma_{\beta}\right|
$$

is the interaction potential among waves of different families.

Let $c>0$ be an arbitrary (small) constant whose precise value will be assumed later. We claim that, for a suitable choice of $\delta_{0}$ and sufficiently small $\|h\|_{\mathbf{L}^{\infty}}$, (depending on $c$ ), the function

$$
t \mapsto \mathcal{S}(t) \doteq V(t)+c \mathcal{Q}(t)
$$

is strictly decreasing at every interaction.

Indeed, assume at a time $t$ two waves with strength $\sigma_{\alpha}$ and $\sigma_{\beta}$ interact at the point $\bar{x}$, and generate two waves of the sizes $\sigma_{h}^{\prime}$ ( $h$-wave) and $\sigma_{p}^{\prime}$ (p-wave). We consider the following cases, with different types of incoming waves.

1. Two neighboring $h$-shocks with strength $\sigma_{\alpha}$ and $\sigma_{\beta}\left(x_{\alpha} \leq x_{\beta}\right)$ interact. By the interaction estimate (3.16), the change in $V$ is

$$
\begin{aligned}
\Delta V & =\left|\sigma_{h}^{\prime}\right|+\left|\sigma_{p}^{\prime}\right|-\left|\sigma_{\alpha}\right|-\left|\sigma_{\beta}\right| \\
& \leq \mathcal{O}(1) \min \left\{\left|P_{l}^{\alpha}-1\right|,\left|P_{l}^{\beta}-1\right|\right\} \cdot\left(\left|\sigma_{\alpha}\right|+\left|\sigma_{\beta}\right|\right) \cdot\left|\sigma_{\alpha} \sigma_{\beta}\right| \\
& =\mathcal{O}(1) \cdot \frac{w_{\alpha, \beta}}{\delta_{0}} \cdot\|h\|_{\mathbf{L}^{\infty}} \cdot\left|\sigma_{\alpha} \sigma_{\beta}\right| .
\end{aligned}
$$


For the change in $\mathcal{Q}$, we see first that $\mathcal{Q}_{h h}$ changes as follows:

$$
\Delta \mathcal{Q}_{h h}=-w_{\alpha, \beta} \cdot\left|\sigma_{\alpha} \sigma_{\beta}\right|+\sum_{\gamma, i_{\gamma}=1}\left\{w_{h, \gamma}\left|\sigma_{h}^{\prime} \sigma_{\gamma}\right|-w_{\alpha, \gamma}\left|\sigma_{\alpha} \sigma_{\gamma}\right|-w_{\beta, \gamma}\left|\sigma_{\beta} \sigma_{\gamma}\right|\right\}
$$

The first negative term is due to interaction, and the second term is due to change in the wave strength after interaction.

Note that the 3 waves $\sigma_{\alpha}, \sigma_{\beta}$ and $\sigma_{h}^{\prime}$ are on the same side of $p=1$. Hence whenever the term $w_{h, \gamma}$ is positive, the other two terms $w_{\alpha, \gamma}, w_{\beta, \gamma}$ are non-zero. Also, note that $w_{h, \gamma}=w_{\alpha, \gamma}$, since the left states of $\sigma_{\alpha}$ and $\sigma_{h}^{\prime}$ are the same.

Hence, the positive terms in the sum of (4.26) are estimated by

$$
\begin{aligned}
w_{h, \gamma}\left|\sigma_{h}^{\prime} \sigma_{\gamma}\right| & -w_{\alpha, \gamma}\left|\sigma_{\alpha} \sigma_{\gamma}\right|-w_{\beta, \gamma}\left|\sigma_{\beta} \sigma_{\gamma}\right| \\
& =w_{\alpha, \gamma} \cdot\left|\sigma_{\gamma}\right| \cdot\left[\left|\sigma_{h}^{\prime}\right|-\left|\sigma_{\alpha}\right|-\left|\sigma_{\beta}\right|\right]+\left(w_{\alpha, \gamma}-w_{\beta, \gamma}\right)\left|\sigma_{\beta} \sigma_{\gamma}\right| .
\end{aligned}
$$

The first term on the r.h.s. can be estimated using again (3.16). To evaluate the quantity $w_{\alpha, \gamma}-w_{\beta, \gamma}$, let $P_{l}^{\alpha}, P_{l}^{\beta}$ be the left state of the waves $\sigma_{\alpha}, \sigma_{\beta}$. Then

$$
\left|w_{\beta, \gamma}-w_{\alpha, \gamma}\right| \leq \delta_{0} \cdot|| P_{l}^{\alpha}-1|-| P_{l}^{\beta}-1||=\delta_{0} \cdot\left|P_{l}^{\alpha}-P_{l}^{\beta}\right|
$$

Since $P_{l}^{\beta}=P_{r}^{\alpha}$, we can use (3.24) and obtain

$$
\left|w_{\beta, \gamma}-w_{\alpha, \gamma}\right| \leq \mathcal{O}(1) \cdot\|h\|_{\mathbf{L}^{\infty}} \cdot w_{\alpha, \beta} \cdot\left|\sigma_{\alpha}\right|
$$

By substituting into (4.27), we deduce

$$
\begin{aligned}
& w_{h, \gamma}\left|\sigma_{h}^{\prime} \sigma_{\gamma}\right|-w_{\alpha, \gamma}\left|\sigma_{\alpha} \sigma_{\gamma}\right|-w_{\beta, \gamma}\left|\sigma_{\beta} \sigma_{\gamma}\right| \\
& \leq w_{\alpha, \gamma} \cdot\left|\sigma_{\gamma}\right| \cdot \mathcal{O}(1) \cdot \frac{w_{\alpha, \beta}}{\delta_{0}} \cdot\|h\|_{\mathbf{L}^{\infty}} \cdot\left|\sigma_{\alpha} \sigma_{\beta}\right|+\mathcal{O}(1) \cdot\|h\|_{\mathbf{L}^{\infty}} \cdot w_{\alpha, \beta} \cdot\left|\sigma_{\alpha}\right| \cdot\left|\sigma_{\beta} \sigma_{\gamma}\right| \\
& \quad=\mathcal{O}(1) \cdot\|h\|_{\mathbf{L}^{\infty}} \cdot w_{\alpha, \beta} \cdot\left|\sigma_{\alpha} \sigma_{\beta}\right| \cdot\left(\frac{w_{\alpha, \gamma}}{\delta_{0}}+1\right)\left|\sigma_{\gamma}\right|
\end{aligned}
$$

Using (4.28), the bound on $P$ and on the total wave strength, then (4.26) is estimated by

$$
\begin{aligned}
\Delta \mathcal{Q}_{h h} & \leq-w_{\alpha, \beta}\left|\sigma_{\alpha} \sigma_{\beta}\right|+\mathcal{O}(1)\|h\|_{\mathbf{L} \infty} w_{\alpha, \beta}\left|\sigma_{\alpha} \sigma_{\beta}\right| \cdot \sum_{\gamma, i_{\gamma}=1}\left\{\frac{w_{\alpha, \gamma}}{\delta_{0}}+1\right\}\left|\sigma_{\gamma}\right| \\
& =-w_{\alpha, \beta} \cdot\left|\sigma_{\alpha} \sigma_{\beta}\right|+\mathcal{O}(1) \cdot\|h\|_{\mathbf{L}^{\infty}} \cdot w_{\alpha, \beta} \cdot\left|\sigma_{\alpha} \sigma_{\beta}\right| .
\end{aligned}
$$

Now, the term $\mathcal{Q}_{p h}+\mathcal{Q}_{p p}$ might increase after interaction, and the change can be roughly estimated as

$$
\begin{aligned}
\Delta \mathcal{Q}_{p h}+\Delta \mathcal{Q}_{p p} & \leq\left(\left|\sigma_{h}^{\prime}\right|-\left|\sigma_{\alpha}\right|-\left|\sigma_{\beta}\right|\right) \sum_{i_{\gamma}=2}\left|\sigma_{\gamma}\right|+\left|\sigma_{p}^{\prime}\right| \cdot \sum_{i_{\gamma}=2}\left|\sigma_{\gamma}\right| \\
& =\mathcal{O}(1) \cdot \frac{w_{\alpha, \beta}}{\delta_{0}} \cdot\|h\|_{\mathbf{L}^{\infty}} \cdot\left|\sigma_{\alpha} \sigma_{\beta}\right| .
\end{aligned}
$$


Notice that all the positive terms are multiplied by the factor $\|h\|_{\mathbf{L}^{\infty}}$. Putting together (4.29) and (4.30), we end up with

$$
\Delta \mathcal{Q} \leq w_{\alpha, \beta} \cdot\left|\sigma_{\alpha} \sigma_{\beta}\right| \cdot\left(-1+\mathcal{O}(1) \cdot \frac{\|h\|_{\mathbf{L}^{\infty}}}{\delta_{0}}+\mathcal{O}(1) \cdot\|h\|_{\mathbf{L}^{\infty}}\right) \leq-\frac{1}{2} w_{\alpha, \beta} \cdot\left|\sigma_{\alpha} \sigma_{\beta}\right|,
$$

where the last inequality holds if $\|h\|_{\mathbf{L}^{\infty}}$ and $\|h\|_{\mathbf{L}^{\infty}} / \delta_{0}$ are sufficiently small. Then, using the estimate (4.25), we finally get

$$
\Delta \mathcal{S}=\Delta V+c \Delta \mathcal{Q} \leq w_{\alpha, \beta} \cdot\left|\sigma_{\alpha} \sigma_{\beta}\right| \cdot\left[-\frac{c}{2}+\mathcal{O}(1) \cdot \frac{1}{\delta_{0}} \cdot\|h\|_{\mathbf{L}^{\infty}}\right] \leq-\frac{c}{4} w_{\alpha, \beta} \cdot\left|\sigma_{\alpha} \sigma_{\beta}\right|
$$

where the last inequality holds by choosing $\|h\|_{\mathbf{L}^{\infty}} /\left(c \delta_{0}\right)$ sufficiently small.

2. Two $h$-waves of opposite sign with strength $\sigma_{\alpha}$ and $\sigma_{\beta}\left(x_{\alpha} \leq x_{\beta}\right)$ interact. They must be one shock and one rarefaction wave. In this case, $V$ is strictly decreasing, because of the cancellation: assuming $\left|\sigma_{\beta}\right|>\left|\sigma_{\alpha}\right|$, we have

$$
\begin{aligned}
\Delta V & =\left|\sigma_{h}^{\prime}\right|+\left|\sigma_{p}^{\prime}\right|-\left|\sigma_{\alpha}\right|-\left|\sigma_{\beta}\right| \\
& \leq\left\{\left|\sigma_{\alpha}+\sigma_{\beta}\right|-\left|\sigma_{\alpha}\right|-\left|\sigma_{\beta}\right|\right\}+\left|\sigma_{h}^{\prime}-\sigma_{\alpha}-\sigma_{\beta}\right|+\left|\sigma_{p}^{\prime}\right| \\
& =-2\left|\sigma_{\alpha}\right|+\mathcal{O}(1) \cdot\|h\|_{\mathbf{L}^{\infty}} \cdot\left|\sigma_{\alpha} \sigma_{\beta}\right| .
\end{aligned}
$$

For the interaction potential, since one of the incoming wave is a rarefaction, we have $w_{\alpha, \beta}=0$, and the change in $\mathcal{Q}_{h h}$ is caused by the change in wave strength at interaction,

$$
\Delta \mathcal{Q}_{h h}=\sum_{\gamma, i_{\gamma}=1}\left\{w_{h, \gamma}^{\prime}\left|\sigma_{h}^{\prime} \sigma_{\gamma}\right|-w_{\alpha, \gamma}\left|\sigma_{\alpha} \sigma_{\gamma}\right|-w_{\beta, \gamma}\left|\sigma_{\beta} \sigma_{\gamma}\right|\right\}
$$

If the outgoing $h$-wave $\sigma_{h}^{\prime}$ is a rarefaction, then $w_{h, \gamma}^{\prime}=0$ and $\Delta \mathcal{Q}_{h h} \leq 0$. If the outgoing $h$-wave is a shock, with strength $\sigma_{h}^{\prime}$, we discuss two cases. First, if $\sigma_{\alpha}$ is a shock and $\sigma_{\beta}$ is a rarefaction, then $w_{\beta, \gamma}=0$ and $w_{h, \gamma}^{\prime}=w_{\alpha, \gamma}$ since they share the same left state of $P$. Due to cancellation, we have $\left|\sigma_{h}^{\prime}\right| \leq\left|\sigma_{\alpha}\right|$, which implies $\Delta \mathcal{Q}_{h h} \leq 0$. Second, if $\sigma_{\beta}$ is a shock and $\sigma_{\alpha}$ is a rarefaction, then $w_{\alpha, \gamma}=0$ and $w_{h, \gamma}^{\prime}=w_{\beta, \gamma}$ because $P_{l}^{\alpha}=P_{l}^{\beta}$ since $\sigma_{\alpha}$ is a rarefaction wave. Furthermore, $\left|\sigma_{h}^{\prime}\right| \leq\left|\sigma_{\beta}\right|$ due to cancellation. Therefore $\Delta \mathcal{Q}_{h h} \leq 0$. The change in $\mathcal{Q}_{p h}+\mathcal{Q}_{p p}$ is estimated in a same way as for the previous case. We have

$$
\Delta \mathcal{Q}_{p h}+\Delta \mathcal{Q}_{p p}=\mathcal{O}(1) \cdot\|h\|_{\mathbf{L}} \cdot\left|\sigma_{\alpha} \sigma_{\beta}\right|
$$

Adding up, the change in $\mathcal{S}$ is

$$
\Delta \mathcal{S} \leq-2\left|\sigma_{\alpha}\right|+(1+c) \cdot \mathcal{O}(1) \cdot\|h\|_{\mathbf{L}^{\infty}} \cdot\left|\sigma_{\alpha} \sigma_{\beta}\right| \leq-\left|\sigma_{\alpha}\right|
$$

where the last inequality holds by choosing $\|h\|_{\mathbf{L}^{\infty}}$ sufficiently small.

3. A $p$-wave $\sigma_{\alpha}$ interacts with a $h$-wave $\sigma_{\beta}$. In this case, by (3.17) the change in $V$ is measured by

$$
\mathcal{O}(1) \cdot\left|\sigma_{\alpha}\right| \cdot\left|\sigma_{\beta}\right| \cdot\|h\|_{\mathbf{L}^{\infty}}
$$


For the interaction potential $\mathcal{Q}$, we have

$$
\Delta \mathcal{Q}_{p h}+\Delta \mathcal{Q}_{p p}=-\left|\sigma_{\alpha}\right| \cdot\left|\sigma_{\beta}\right|+\mathcal{O}(1) \cdot\left|\sigma_{\alpha} \sigma_{\beta}\right| \cdot\|h\|_{\mathbf{L}^{\infty}},
$$

where the first negative term is due to interaction, and the last term is due to the change of wave strength after interaction. For the term $\mathcal{Q}_{h h}$, we have

$$
\Delta \mathcal{Q}_{h h}=\sum_{\gamma, i_{\gamma}=1}\left\{w_{h, \gamma}^{\prime}\left|\sigma_{h}^{\prime} \sigma_{\gamma}\right|-w_{\beta, \gamma}\left|\sigma_{\beta} \sigma_{\gamma}\right|\right\}
$$

To see how $\mathcal{Q}_{h h}$ changes, we consider two cases. First, if the $p$-wave $\sigma_{\alpha}$ crossed $p=1$, then the two waves $\sigma_{\beta}$ and $\sigma_{h}^{\prime}$ must be of different type, one shock and one rarefaction. If $\sigma_{h}^{\prime}$ is a rarefaction, then $w_{h, \gamma}^{\prime}=0$ and $\Delta \mathcal{Q}_{h h} \leq 0$. Otherwise, if $\sigma_{h}^{\prime}$ is a shock, then $w_{\beta, \gamma}=0$. Let $P_{l}^{\alpha}, P_{r}^{\alpha}$ be the left, right state of $\sigma_{\alpha}$, we have

$$
w_{h, \gamma}^{\prime}=\delta_{0} \min \left\{\left|P_{l}^{\alpha}-1\right|,\left|P_{l}^{\gamma}-1\right|\right\} \leq \delta_{0}\left|\sigma_{\alpha}\right|,
$$

and we have the estimate

$$
\Delta \mathcal{Q}_{h h} \leq \sum_{\gamma, i_{\gamma}=1} w_{h, \gamma}^{\prime}\left|\sigma_{h}^{\prime} \sigma_{\gamma}\right| \leq \mathcal{O}(1) \delta_{0} \cdot\left|\sigma_{\alpha}\right| \cdot\left[\left|\sigma_{\beta}\right|+\mathcal{O}(1)\|h\|_{\mathbf{L} \infty}\left|\sigma_{\alpha} \sigma_{\beta}\right|\right] \leq \mathcal{O}(1) \delta_{0} \cdot\left|\sigma_{\alpha} \sigma_{\beta}\right| .
$$

For the second case, when $\sigma_{\alpha}$ does not cross $p=1$, then the weights $w_{h, \gamma}^{\prime}$ and $w_{\beta, \gamma} \neq 0$ will be either both zero or both non-zero, and

$$
w_{h, \gamma}^{\prime}-w_{\beta, \gamma} \leq \delta_{0}\left\{\left|P_{l}^{\alpha}-1\right|-\left|P_{r}^{\alpha}-1\right|\right\} \leq \delta_{0}\left|P_{l}^{\alpha}-P_{r}^{\alpha}\right|=\delta_{0}\left|\sigma_{\alpha}\right| .
$$

Then, we have

$$
\begin{aligned}
\Delta \mathcal{Q}_{h h} & =\sum_{\gamma, i_{\gamma}=1} w_{h, \gamma}^{\prime}\left(\left|\sigma_{h}^{\prime}\right|-\left|\sigma_{\beta}\right|\right)\left|\sigma_{\gamma}\right|+\sum_{\gamma, i_{\gamma}=1}\left(w_{h, \gamma}^{\prime}-w_{\beta, \gamma}\right)\left|\sigma_{\beta} \sigma_{\gamma}\right| \\
& \leq \mathcal{O}(1) \cdot \delta_{0}\left|\sigma_{\alpha} \sigma_{\beta}\right| .
\end{aligned}
$$

In conclusion, we have

$$
\Delta \mathcal{Q} \leq-\left|\sigma_{\alpha} \sigma_{\beta}\right|+\mathcal{O}(1) \cdot\left|\sigma_{\alpha} \sigma_{\beta}\right| \cdot\left(\delta_{0}+\|h\|_{\mathbf{L}^{\infty}}\right) \leq-\frac{1}{2}\left|\sigma_{\alpha} \sigma_{\beta}\right|
$$

where the last inequality holds for $\delta_{0}>0$ sufficiently small. Therefore

$$
\Delta \mathcal{S}=\Delta V+c \Delta \mathcal{Q} \leq\left|\sigma_{\alpha} \sigma_{\beta}\right|\left(-\frac{c}{2}+\mathcal{O}(1)\|h\|_{\mathbf{L}^{\infty}}\right) \leq-\frac{c}{4}\left|\sigma_{\alpha} \sigma_{\beta}\right|,
$$

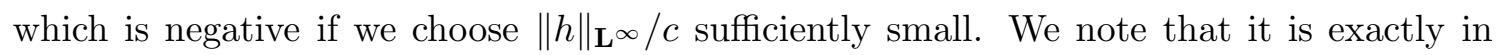
this estimate that it requires to choose the constant $\delta_{0}>0$ sufficiently small.

4. Two $p$-waves interact. Similarly, by (3.14), the change in wave strength $V$ is measured by

$$
\mathcal{O}(1) \cdot\left|\sigma_{\alpha}\right| \cdot\left|\sigma_{\beta}\right| \cdot\|h\|_{\mathbf{L}^{\infty}}
$$

and the change in the interaction potential $\mathcal{Q}$ is measured by

$$
-\left|\sigma_{\alpha}\right| \cdot\left|\sigma_{\beta}\right|+\mathcal{O}(1) \cdot\left|\sigma_{\alpha}\right| \cdot\left|\sigma_{\beta}\right| \cdot\|h\|_{\mathbf{L}^{\infty}} .
$$

Add up these two terms, we get the change in $\mathcal{S}$,

$$
\Delta \mathcal{S}=-c\left|\sigma_{\alpha} \sigma_{\beta}\right|+(1+c) \mathcal{O}(1) \cdot\left|\sigma_{\alpha} \sigma_{\beta}\right| \cdot\|h\|_{\mathbf{L}^{\infty}} \leq-\frac{c}{2}\left|\sigma_{\alpha} \sigma_{\beta}\right|,
$$

if $\|h\|_{\mathbf{L}^{\infty}}$ is sufficiently small. 


\subsection{New total wave strength and interaction potential with the source term}

The analysis in the previous step was valid in connection with (3.1), without the source term. To handle the original system (1.2), we need to account for the possible increase in wave strengths, due to the source terms. By (3.4), the size of an $h$-jump grows at a rate proportional to the amount of $|p-1|$-mass it crosses. Similarly, by (3.5), the size of a $p$-jump grows at a rate proportional to the amount of $h$-mass it crosses. It is thus natural to consider a weight functional $Z=Z(t, x)$ measuring how much mass a given front located at $x$ still has to cross. Recalling (4.4) and (4.11)-(4.12), we thus define

$$
Z(t, x) \doteq V^{h}(t, x) \cdot V^{p}(t, x)
$$

where $V^{h}$ and $V^{p}$ are defined in (4.11) and (4.12), for suitable constants $\kappa_{1}>0$ and $\kappa_{2}>0$.

We first compute the derivative of the functional $Z$ along a differentiable curve $t \mapsto x(t)$ : using (4.13)-(4.14), we have

$$
\begin{aligned}
\frac{d}{d t} Z(t, x(t)) & =\frac{d}{d t}\left(V^{h}(t, x(t))\right) \cdot V^{p}+V^{h} \cdot \frac{d}{d t}\left(V^{p}(t, x(t))\right) \\
& \leq-\kappa_{1} V^{h}(h-\dot{x})|p-1| V^{p}-V^{h} \cdot \kappa_{2} V^{p}(p+\dot{x}) W h \\
& =-Z(t, x(t)) \cdot\left\{\kappa_{1}(h-\dot{x})|p-1|+\kappa_{2}(p+\dot{x}) W h\right\} .
\end{aligned}
$$

Note that the previous quantity is $\leq 0$ if $-p \leq \dot{x} \leq h$.

The presence of source terms can now be accounted by redefining the quantities (4.19)(4.23), replacing each wave strength $\left|\sigma_{\alpha}\right|$ with the weighted strength $Z\left(x_{\alpha}\right)\left|\sigma_{\alpha}\right|$. More precisely we define, for every time $t$ at which no interactions occur,

$$
\widetilde{\mathcal{S}}=\widetilde{V}+c \widetilde{\mathcal{Q}},
$$

where

$$
\widetilde{V}=\sum_{\alpha} Z\left(x_{\alpha}\right)\left|\sigma_{\alpha}\right|, \quad \widetilde{\mathcal{Q}}=\widetilde{\mathcal{Q}}_{h h}+\widetilde{\mathcal{Q}}_{p p}+\widetilde{\mathcal{Q}}_{p h}
$$

and

$$
\begin{aligned}
\widetilde{\mathcal{Q}}_{h h} & =\sum_{i_{\alpha}=i_{\beta}=1, x_{\alpha}<x_{\beta}} w_{\alpha, \beta} Z\left(x_{\alpha}\right)\left|\sigma_{\alpha}\right| Z\left(x_{\beta}\right)\left|\sigma_{\beta}\right|, \\
\widetilde{\mathcal{Q}}_{p p} & =\sum_{(\alpha, \beta) \in \mathcal{A}_{2}} Z\left(x_{\alpha}\right)\left|\sigma_{\alpha}\right| Z\left(x_{\beta}\right)\left|\sigma_{\beta}\right|, \\
\widetilde{\mathcal{Q}}_{p h} & =\sum_{i_{\alpha}=2, i_{\beta}=1, x_{\alpha}<x_{\beta}} Z\left(x_{\beta}\right)\left|\sigma_{\alpha}\right| Z\left(x_{\beta}\right)\left|\sigma_{\beta}\right| .
\end{aligned}
$$

We now observe that, at times where two wave-fronts interact, the same arguments as in subsection 4.4 show that the quantities $\widetilde{\mathcal{S}}$ is decreasing. Indeed, the presence of the weights $Z\left(x_{\alpha}\right)$ only increases the sizes of the coefficients $\mathcal{O}(1)$ in the various estimates. This can be counter-balanced by choosing $\|h\|_{\mathbf{L}^{\infty}}$ sufficiently small. We omit the details.

\subsection{The bounds on the total variations of $h$ and $p$.}

Next, consider a time $t_{k}$ where the functions $h, p$ are redefined as in (3.2), accounting for the source terms. We claim that the weighted functional $\widetilde{\mathcal{S}}=\widetilde{V}+c \widetilde{\mathcal{Q}}$ is decreasing from $t=t_{k-1}+$ to $t=t_{k}+$, for suitable choice of $c$ (apart from small terms due to wave interactions). 


\subsubsection{The decrease of $\widetilde{V}$.}

Indeed, consider a wave-front of the first family, located at $x_{\alpha}(\cdot)$ with size $\sigma_{h}$, and let $\left(h_{l}, p_{l}\right)$ and $\left(h_{r}, p_{r}\right)$ be its left and right states, respectively. Assume that this front does not interact with other fronts during the interval $\left(t_{k-1}, t_{k}\right)$. At time $t_{k}$, this front is replaced by two fronts of strengths $\sigma_{h}^{+}, \sigma_{p}^{+}$, whose strength is estimated by (3.4).

Following (4.33), we have

$$
\frac{d}{d t} Z\left(t, x_{\alpha}(t)\right) \leq-Z\left(t, x_{\alpha}(t)\right)\left\{\kappa_{1}\left(h_{l}-\dot{x}_{\alpha}\right)\left|p_{l}-1\right|+\kappa_{2}\left(p_{r}+\dot{x}_{\alpha}\right) W\left(t, x_{\alpha}(t)\right) h_{r}\right\} .
$$

Note that the first term comes from differentiation of $V^{h}$, which by definition (4.11) includes the integral over the interval $(-\infty, x)$, therefore $h, p$ is evaluated at the left state. In the meanwhile, the second term comes from differentiation of $V^{p}$, which by definition (4.12) includes the integral over the interval $(x, \infty)$, therefore $h, p$ is evaluated at the right state.

According to (2.4), the speed $\dot{x}_{\alpha}$ is estimated by

$$
\dot{x}_{\alpha}=\lambda_{1}\left(h_{l}, p_{l}\right)+\mathcal{O}(1)\left|\sigma_{h}\right| \cdot\left|p_{l}-1\right|=-p_{l}+\mathcal{O}(1) \cdot h_{l} \cdot\left|p_{l}-1\right|+\mathcal{O}(1)\left|\sigma_{h}\right| \cdot\left|p_{l}-1\right|,
$$

and hence

$$
h_{l}-\dot{x}_{\alpha} \geq c_{0}>0
$$

for some positive constant $c_{0}$. On the other hand, the quantity $p_{r}+\dot{x}_{\alpha}$ may not have a specific sign. We can estimate it as follows:

$$
\begin{aligned}
\left|p_{r}+\dot{x}_{\alpha}\right| & =\left|p_{r}+\lambda_{1}\left(h_{r}, p_{r}\right)+\mathcal{O}(1) \sigma_{h}\left(p_{r}-1\right)\right| \\
& \leq \mathcal{O}(1) \cdot h_{r}\left|p_{r}-1\right|+\mathcal{O}(1)\left|\sigma_{h}\right| \cdot\left|p_{r}-1\right| \\
& =\mathcal{O}(1) \cdot\left(h_{r}+\left|\sigma_{h}\right|\right)\left|p_{l}-1\right|
\end{aligned}
$$

since $p_{l}-1$ and $p_{r}-1$ are of the same order of magnitude. Using (4.40) and (4.41) into (4.39), and recalling that the weights $W$ can be large but are uniformly bounded, we obtain

$$
\frac{d}{d t} Z\left(t, x_{\alpha}(t)\right) \leq Z\left(t, x_{\alpha}(t)\right) \cdot\left(-\kappa_{1} c_{0}\left|p_{l}-1\right|+\kappa_{2} \cdot \mathcal{O}(1) \cdot\left(h_{r}+\left|\sigma_{h}\right|\right) h_{r}\left|p_{l}-1\right|\right) .
$$

Finally, by choosing $\kappa_{1}$ sufficiently large, and the norm $\|h\|_{\mathbf{L}^{\infty}}$ sufficiently small, such that $\mathcal{O}(1) \kappa_{2}\left(\|h\|_{\mathbf{L}^{\infty}}\right)^{2} \leq \frac{1}{2} \kappa_{1} c_{0}$, we conclude that

$$
\frac{d}{d t} Z\left(t, x_{\alpha}(t)\right) \leq-Z\left(t, x_{\alpha}(t)\right) \cdot \kappa_{1} \frac{c_{0}}{2}\left|p_{l}-1\right| .
$$

By integrating the previous inequality on $\left(t_{k-1}, t_{k}\right)$, we get the following estimate on $Z$,

$$
\begin{aligned}
& Z\left(t_{k}, x_{\alpha}\left(t_{k}\right)\right) \cdot\left(\left|\sigma_{h}^{+}\right|+\left|\sigma_{p}^{+}\right|\right) \\
& \quad \leq Z\left(t_{k-1}, x_{\alpha}\left(t_{k-1}\right)\right) \cdot\left|\sigma_{h}\right| \cdot\left(1-\kappa_{1} \Delta t \cdot \frac{c_{0}}{4}\left|p_{l}-1\right|\right)\left(1+\mathcal{O}(1) \cdot \Delta t \cdot\left|p_{l}-1\right|\right) .
\end{aligned}
$$

Choosing the time step $\Delta t$ small enough and $\kappa_{1}$ large enough, such that

$$
\kappa_{1} \frac{c_{0}}{4}-\mathcal{O}(1)+\mathcal{O}(1) \cdot \kappa_{1} \Delta t \frac{c_{0}}{4}\left|p_{l}-1\right| \geq c_{1}>0
$$


for some positive constant $c_{1}$, we get

$$
Z\left(t_{k}, x_{\alpha}\left(t_{k}\right)\right) \cdot\left(\left|\sigma_{h}^{+}\right|+\left|\sigma_{p}^{+}\right|\right) \leq Z\left(t_{k-1}, x_{\alpha}\left(t_{k-1}\right)\right)\left|\sigma_{h}\right|\left(1-c_{1}\left|p_{l}-1\right| \Delta t\right) .
$$

Next, consider a wave-front of the second family, located at $x_{\beta}(\cdot)$ with size $\sigma_{p}$. We still assume no interaction during the time interval $\left(t_{k-1}, t_{k}\right)$.

As before, we evaluate the quantities $\left(h_{l}-\dot{x}_{\beta}\right)$ and $\left(p_{r}+\dot{x}_{\beta}\right)$. Since here $\dot{x}_{\beta} \sim h$, the second quantity will be significant and the first one negligible. Indeed, according to the second equation in $(2.4)$, we get

$$
\begin{aligned}
p_{r}+\dot{x}_{\beta} & =p_{r}+\lambda_{2}\left(h_{r}, p_{r}\right)+\mathcal{O}(1)\left|\sigma_{p}\right| h_{r} \\
& =p_{r}+h_{r}+\mathcal{O}(1)\left(\left|p_{r}-1\right|+\left|\sigma_{p}\right|\right) h_{r} \\
& \geq \bar{c}_{0}>0
\end{aligned}
$$

for some positive constant $\bar{c}_{0}$ (by choosing $\|h\|_{\mathbf{L}^{\infty}}$ sufficiently small), and

$$
h_{l}-\dot{x}_{\beta}=h_{l}-\lambda_{2}\left(h_{l}, p_{l}\right)+\mathcal{O}(1)\left|\sigma_{p}\right| h_{l}=\mathcal{O}(1)\left(\left|p_{l}-1\right|+\left|\sigma_{p}\right|\right) h_{r},
$$

since $h_{r}, h_{l}$ are of the same order of magnitude.

Now, by (4.43)-(4.44), and recalling that $W \geq 1$, we have the following estimate on $Z$ :

$$
\begin{aligned}
\frac{d}{d t} Z\left(t, x_{\beta}(t)\right) & \leq-Z\left(t, x_{\beta}(t)\right) \cdot\left\{\kappa_{1}\left(h_{l}-\dot{x}_{\beta}\right)\left|p_{l}-1\right|+\kappa_{2}\left(p_{r}+\dot{x}_{\beta}\right) W h_{r}\right\} \\
& \leq Z\left(t, x_{\beta}(t)\right) \cdot h_{r} \cdot\left(-\kappa_{2} \bar{c}_{0}+\mathcal{O}(1) \kappa_{1}\left|p_{l}-1\right|\left(\left|p_{l}-1\right|+\left|\sigma_{p}\right|\right)\right) .
\end{aligned}
$$

Finally, by choosing $\kappa_{2}$ sufficiently larger than $\kappa_{1}$, such that

$$
\kappa_{1} \mathcal{O}(1)\left|p_{l}-1\right|\left(\left|p_{l}-1\right|+\left|\sigma_{p}\right|\right) \leq \kappa_{2} \frac{\bar{c}_{0}}{2}
$$

we conclude

$$
\frac{d}{d t} Z\left(t, x_{\beta}(t)\right) \leq-Z\left(t, x_{\beta}(t)\right) \cdot \kappa_{2} \frac{\bar{c}_{0}}{2} h_{r} .
$$

Therefore, neglecting the interaction terms and using (3.5), we have

$$
\begin{aligned}
& Z\left(t_{k}, x_{\beta}\left(t_{k}\right)\right) \cdot\left(\left|\sigma_{h}^{+}\right|+\left|\sigma_{p}^{+}\right|\right) \\
& \quad \leq Z\left(t_{k-1}, x_{\beta}\left(t_{k-1}\right)\right) \cdot\left|\sigma_{p}\right| \cdot\left[1-\kappa_{2} \Delta t \cdot \frac{\bar{c}_{0}}{4} h_{r}\right]\left(1+\mathcal{O}(1) \cdot \Delta t \cdot h_{r}\right) .
\end{aligned}
$$

Again, we can choose $\kappa_{2}$ sufficiently large, such that

$$
\kappa_{2} \frac{\bar{c}_{0}}{4}-\mathcal{O}(1)+\mathcal{O}(1) \kappa_{2} \Delta t \cdot \frac{\bar{c}_{0}}{4} h_{r} \geq c_{2}>0
$$

for some positive constant $c_{2}$, we have the following estimate

$$
Z\left(t_{k}, x_{\beta}\left(t_{k}\right)\right) \cdot\left(\left|\sigma_{h}^{+}\right|+\left|\sigma_{p}^{+}\right|\right) \leq Z\left(t_{k-1}, x_{\beta}\left(t_{k-1}\right)\right) \cdot\left|\sigma_{p}\right| \cdot\left(1-c_{2} h_{r} \Delta t\right) .
$$

We conclude that, in both cases (4.42) and (4.45), the weighted strength of all jumps decreases from $t_{k-1}$ to $t_{k}$, as follows:

$$
\Delta \widetilde{V} \leq-c_{1} \Delta t \sum_{\alpha, i_{\alpha}=1} Z\left(x_{\alpha}\left(t_{k-1}\right)\right)\left|\sigma_{\alpha}\right|\left|p_{l}^{\alpha}-1\right|-c_{2} \Delta t \sum_{\beta, i_{\beta}=2} Z\left(x_{\beta}\left(t_{k-1}\right)\right)\left|\sigma_{\beta}\right| h_{r}^{\beta} .
$$




\subsubsection{The change of $\widetilde{\mathcal{Q}}$.}

Now we study the change in the $\widetilde{\mathcal{Q}}$ term from time $t_{k-1}$ to $t_{k}$. Given a pair of waves, we have 4 possible cases, according to the families of the two waves. For each case we evaluate the variation of the corresponding terms in $\widetilde{\mathcal{Q}}$ from time $t_{k-1}$ to $t_{k}$.

Case 1. Assume at $t_{k-1}$ we have two waves of the first family, located at $x_{\alpha}$ and $\tilde{x}_{\alpha}$, with wave strengths $\sigma_{\alpha}$ and $\tilde{\sigma}_{\alpha}$. At time $t_{k}$, after the fractional step (3.2), each 1-wave will become two waves, one from each family, with strengths $\left(\sigma_{\alpha}^{+}, \sigma_{\beta}^{+}\right)$and $\left(\tilde{\sigma}_{\alpha}^{+}, \tilde{\sigma}_{\beta}^{+}\right)$located at $x_{\alpha}^{+}$and $\tilde{x}_{\alpha}^{+}$, respectively. See figure 3 for an illustration.

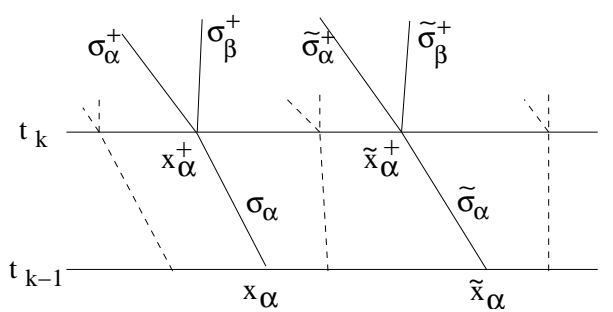

Figure 3: Case 1, two 1-waves $\sigma_{\alpha}$ and $\tilde{\sigma}_{\alpha}$ from time $t_{k-1}$ to $t_{k}$. Here the dotted lines denote other wave fronts which are not being considered in this case.

To simplify notation we introduce $Z_{\alpha}=Z\left(t_{k-1}, x_{\alpha}\right), Z_{\alpha}^{+}=Z\left(t_{k}, x_{\alpha}^{+}\right)$and similarly for $\tilde{Z}_{\alpha}$, $\tilde{Z}_{\alpha}^{+}$. The change in $\widetilde{\mathcal{Q}}_{h h}$ related to this pair of waves can be estimated as

$$
\Delta \widetilde{\mathcal{Q}}_{h h}=w_{\alpha^{+}, \tilde{\alpha}^{+}} Z_{\alpha}^{+}\left|\sigma_{\alpha}^{+}\right| \cdot \tilde{Z}_{\alpha}^{+}\left|\tilde{\sigma}_{\alpha}^{+}\right|-w_{\alpha, \tilde{\alpha}} Z_{\alpha}\left|\sigma_{\alpha}\right| \cdot \tilde{Z}_{\alpha}\left|\tilde{\sigma}_{\alpha}\right| \doteq(I)+(I I),
$$

where

$$
(I) \doteq w_{\alpha, \tilde{\alpha}}\left\{Z_{\alpha}^{+}\left|\sigma_{\alpha}^{+}\right| \cdot \tilde{Z}_{\alpha}^{+}\left|\tilde{\sigma}_{\alpha}^{+}\right|-Z_{\alpha}\left|\sigma_{\alpha}\right| \cdot \tilde{Z}_{\alpha}\left|\tilde{\sigma}_{\alpha}\right|\right\}
$$

and

$$
(I I) \doteq\left\{w_{\alpha^{+}, \tilde{\alpha}^{+}}-w_{\alpha, \tilde{\alpha}}\right\} Z_{\alpha}^{+}\left|\sigma_{\alpha}^{+}\right| \cdot \tilde{Z}_{\alpha}^{+}\left|\tilde{\sigma}_{\alpha}^{+}\right| \cdot
$$

We first note that, after the fractional step, each 1-wave remains on the same side of $p=1$ and does not change type. Then, in the above formula, $w_{\alpha^{+}, \tilde{\alpha}^{+}} \neq 0$ if and only if $w_{\alpha, \tilde{\alpha}} \neq 0$.

If $w_{\alpha, \tilde{\alpha}}=0$, i.e, $\sigma_{\alpha}$ and $\tilde{\sigma}_{\alpha}$ on the opposite sides of the line $p=1$, we trivially have $\Delta \widetilde{\mathcal{Q}}_{h h}=0$. We need to consider the case when $w_{\alpha, \tilde{\alpha}} \neq 0$. The first term $(I)$ is less than 0 thanks to (4.42):

$$
(I) \leq w_{\alpha, \tilde{\alpha}} Z_{\alpha} \tilde{Z}_{\alpha} \cdot\left|\sigma_{\alpha} \tilde{\sigma}_{\alpha}\right|\left\{\left(1-c_{1}\left|p_{l}-1\right| \Delta t\right)\left(1-c_{1}\left|\tilde{p}_{l}-1\right| \Delta t\right)-1\right\} \leq 0 .
$$

For the second term $(I I)$, we need an estimate on the change of $P$ due to the source term step (3.2). First we see if $P_{l}<1, \tilde{P}_{l}<1$ then after the time step (3.2) $h$ will decrease. From the geometry of the integral curves we see that $P$ will increase, and therefore we have $P_{l}^{+} \geq P_{l}$ and $\tilde{P}_{l}^{+} \geq \tilde{P}_{l}$. This gives $w_{\alpha^{+}, \tilde{\alpha}^{+}} \leq w_{\alpha, \tilde{\alpha}}$, and therefore we have $(I I) \leq 0$.

Otherwise, if $P_{l}>1, \tilde{P}_{l}>1$, after time step (3.2) $h$ will increase and so will $P$, and we have $P_{l}^{+} \geq P_{l}$ and $\tilde{P}_{l}^{+} \geq \tilde{P}_{l}$, and the increments can be bounded by

$$
P_{l}^{+}-P_{l} \leq \mathcal{O}(1) \Delta t h_{l}\left|p_{l}-1\right|, \quad \tilde{P}_{l}^{+}-\tilde{P}_{l} \leq \mathcal{O}(1) \Delta t \tilde{h}_{l}\left|\tilde{p}_{l}-1\right|,
$$


which is equivalent to

$$
P_{l}^{+}-1 \leq P_{l}-1+\mathcal{O}(1) \Delta t h_{l}\left|p_{l}-1\right|, \quad \tilde{P}_{l}^{+}-1 \leq \tilde{P}_{l}-1+\mathcal{O}(1) \Delta t \tilde{h}_{l}\left|\tilde{p}_{l}-1\right| .
$$

Hence we get

$$
\min \left\{\left|P_{l}^{+}-1\right|,\left|\tilde{P}_{l}^{+}-1\right|\right\}-\min \left\{\left|P_{l}-1\right|,\left|\tilde{P}_{l}-1\right|\right\}=\mathcal{O}(1) \Delta t \cdot\|h\|_{\mathbf{L}^{\infty}} \cdot\left|P_{l}-1\right| .
$$

Therefore, we have

$$
\Delta \widetilde{\mathcal{Q}}_{h h} \leq(I I) \leq \mathcal{O}(1) \delta_{0} \cdot \Delta t \cdot\|h\|_{\mathbf{L}^{\infty}} \cdot\left|P_{l}-1\right| \cdot Z_{\alpha} \tilde{Z}_{\alpha}\left|\sigma_{\alpha} \tilde{\sigma}_{\alpha}\right| .
$$

The terms $\widetilde{\mathcal{Q}}_{p p}$ and $\widetilde{\mathcal{Q}}_{p h}$ may both increase. By (3.4) and (4.42), the increment is small, estimated as

$$
\Delta \widetilde{\mathcal{Q}}_{p h}+\Delta \widetilde{\mathcal{Q}}_{p p}=Z_{\alpha}^{+}\left|\sigma_{\beta}^{+}\right| \cdot \tilde{Z}_{\alpha}^{+}\left(\left|\tilde{\sigma}_{\alpha}^{+}\right|+\left|\tilde{\sigma}_{\beta}^{+}\right|\right) \leq \mathcal{O}(1) \cdot Z_{\alpha} \tilde{Z}_{\alpha}\left|\sigma_{\alpha} \tilde{\sigma}_{\alpha}\right| \Delta t\left|p_{l}-1\right| .
$$

Adding together the bounds on $\Delta \widetilde{\mathcal{Q}}_{h h}, \Delta \widetilde{\mathcal{Q}}_{p h}$ and $\Delta \widetilde{\mathcal{Q}}_{p p}$, we get

$$
\Delta \widetilde{\mathcal{Q}} \leq \mathcal{O}(1) \cdot Z_{\alpha} \tilde{Z}_{\alpha}\left|\sigma_{\alpha} \tilde{\sigma}_{\alpha}\right| \cdot \Delta t \cdot\left|P_{l}-1\right| .
$$

Case 2. Assume at $t_{k-1}$ we have two waves from of the second family, located at $x_{\beta}$ and $\tilde{x}_{\beta}$, with wave strengths $\sigma_{\beta}$ and $\tilde{\sigma}_{\beta}$. At time $t_{k}$, after the fractional step (3.2), each 2-wave will become two waves, one from each family, with strengths $\left(\sigma_{\alpha}^{+}, \sigma_{\beta}^{+}\right)$and $\left(\tilde{\sigma}_{\alpha}^{+}, \tilde{\sigma}_{\beta}^{+}\right)$located at $x_{\beta}^{+}$ and $\tilde{x}_{\beta}^{+}$, respectively. See figure 4 for an illustration. As in Case 1 , we introduce the notation $Z_{\beta}=Z\left(t_{k-1}, x_{\beta}\right), Z_{\beta}^{+}=Z\left(t_{k}, x_{\beta}^{+}\right)$and similarly $\tilde{Z}_{\beta}, \tilde{Z}_{\beta}^{+}$.

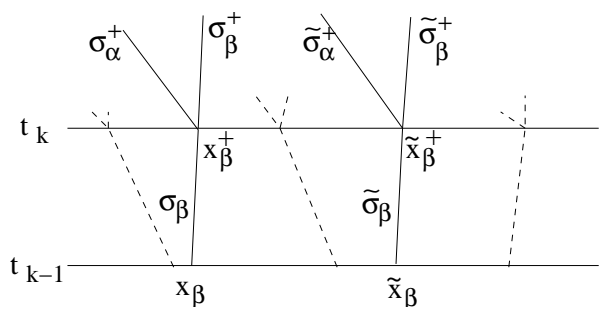

Figure 4: Case 2, two 2-waves $\sigma_{\beta}$ and $\tilde{\sigma}_{\beta}$ from time $t_{k-1}$ to $t_{k}$. Here the dotted lines denote other wave fronts which are not being considered in this case.

Using (4.45), the changes in $\widetilde{\mathcal{Q}}_{p p}, \widetilde{\mathcal{Q}}_{p h}$ are estimated as

$$
\begin{aligned}
\Delta \widetilde{\mathcal{Q}}_{p p}+\Delta \widetilde{\mathcal{Q}}_{p h} & =-Z_{\beta}\left|\sigma_{\beta}\right| \cdot \tilde{Z}_{\beta}\left|\tilde{\sigma}_{\beta}\right|+Z_{\beta}^{+}\left|\sigma_{\beta}^{+}\right| \cdot \tilde{Z}_{\beta}^{+}\left(\left|\tilde{\sigma}_{\alpha}^{+}\right|+\left|\tilde{\sigma}_{\beta}^{+}\right|\right) \\
& \leq Z_{\beta} \tilde{Z}_{\beta}\left|\sigma_{\beta} \tilde{\sigma}_{\beta}\right| \cdot\left\{-c_{2} \Delta t\left(h_{r}+\tilde{h}_{r}\right)+c_{2}^{2} h_{r} \tilde{h}_{r}(\Delta t)^{2}\right\}
\end{aligned}
$$

while for $\widetilde{\mathcal{Q}}_{h h}$ we use (3.5) to get

$$
\begin{aligned}
\Delta \widetilde{\mathcal{Q}}_{h h} & =w_{\alpha^{+}, \tilde{\alpha}^{+}} \cdot Z_{\beta}^{+}\left|\sigma_{\alpha}^{+}\right| \cdot \tilde{Z}_{\beta}^{+}\left|\tilde{\sigma}_{\alpha}^{+}\right| \leq \mathcal{O}(1) \cdot Z_{\beta}\left|\sigma_{\beta}\right| h_{l} \Delta t \cdot \tilde{Z}_{\beta}\left|\tilde{\sigma}_{\beta}\right| \tilde{h}_{l} \Delta t \\
& \leq \mathcal{O}(1) \cdot Z_{\beta} \tilde{Z}_{\beta}\left|\sigma_{\beta} \tilde{\sigma}_{\beta}\right| \cdot h_{r} \tilde{h}_{r} \cdot(\Delta t)^{2} .
\end{aligned}
$$

Adding them together, we have

$$
\Delta \widetilde{\mathcal{Q}} \leq Z_{\beta} \tilde{Z}_{\beta}\left|\sigma_{\beta} \tilde{\sigma}_{\beta}\right| \cdot \Delta t \cdot\left\{-c_{2}\left(h_{r}+\tilde{h}_{r}\right)+c_{2}^{2} h_{r} \tilde{h}_{r} \Delta t+\mathcal{O}(1) \cdot h_{r} \tilde{h}_{r} \cdot \Delta t\right\} .
$$

By choosing $\Delta t$ or $\|h\|_{\mathbf{L}^{\infty}}$ small enough, we conclude that $\widetilde{\mathcal{Q}}$ decreases. 
Case 3. We now consider the case where we have two approaching waves at $t_{k-1}$, a 2 -wave of strength $\sigma_{\beta}$ at $x_{\beta}$ and a 1-wave of strength $\tilde{\sigma}_{\alpha}$ at $\tilde{x}_{\alpha}$, with $x_{\beta}<\tilde{x}_{\alpha}$. At time $t_{k}$, after the fractional step (3.2), each wave will become two waves, one from each family, with strengths $\left(\sigma_{\alpha}^{+}, \sigma_{\beta}^{+}\right)$and $\left(\tilde{\sigma}_{\alpha}^{+}, \tilde{\sigma}_{\beta}^{+}\right)$located at $x_{\beta}^{+}$and $\tilde{x}_{\alpha}^{+}$, respectively. See figure 5 for an illustration.

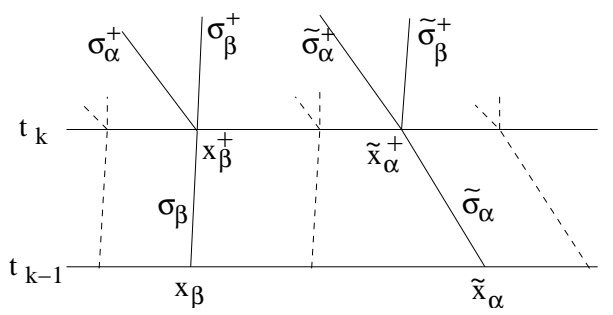

Figure 5: Case 3, a 2-wave $\sigma_{\beta}$ and a 1 -wave $\tilde{\sigma}_{\alpha}$ approaching each other at $t_{k-1}$. Here the dotted lines denote other wave fronts which are not being considered in this case.

Using (4.42) and (4.45) we get

$$
\begin{aligned}
\Delta \widetilde{\mathcal{Q}}_{p p}+\Delta \widetilde{\mathcal{Q}}_{p h} & =-Z_{\beta}\left|\sigma_{\beta}\right| \cdot \tilde{Z}_{\alpha}\left|\tilde{\sigma}_{\alpha}\right|+Z_{\beta}^{+}\left|\sigma_{\beta}^{+}\right| \cdot \tilde{Z}_{\alpha}^{+}\left(\left|\tilde{\sigma}_{\alpha}^{+}\right|+\left|\tilde{\sigma}_{\beta}^{+}\right|\right) \\
& \leq Z_{\beta}\left|\sigma_{\beta}\right| \cdot \tilde{Z}_{\alpha}\left|\tilde{\sigma}_{\alpha}\right| \cdot\left\{-1+\left(1-c_{1}\left|\tilde{p}_{l}-1\right| \Delta t\right)\left(1-c_{2} h_{r} \Delta t\right)\right\} \\
& \leq Z_{\beta}\left|\sigma_{\beta}\right| \cdot \tilde{Z}_{\alpha}\left|\tilde{\sigma}_{\alpha}\right|\left\{-\Delta t \cdot\left(c_{1}\left|\tilde{p}_{l}-1\right|+c_{2} h_{r}\right)+c_{1} c_{2}\left|\tilde{p}_{l}-1\right| h_{r}(\Delta t)^{2}\right\},
\end{aligned}
$$

and by (3.5) we get

$$
\begin{aligned}
\Delta \widetilde{\mathcal{Q}}_{h h} & =w_{\alpha^{+}, \tilde{\alpha}^{+}} \cdot Z_{\beta}^{+}\left|\sigma_{\alpha}^{+}\right| \cdot \tilde{Z}_{\alpha}^{+}\left|\tilde{\sigma}_{\alpha}^{+}\right| \\
& \leq \mathcal{O}(1) \cdot \min \left\{\left|p_{l}-1\right|,\left|\tilde{p}_{l}-1\right|\right\} \cdot Z_{\beta}\left|\sigma_{\beta}\right| \cdot \tilde{Z}_{\alpha}\left|\tilde{\sigma}_{\alpha}\right| \cdot h_{r} \cdot \Delta t .
\end{aligned}
$$

Summing up the previous inequalities, we get

$$
\Delta \widetilde{\mathcal{Q}} \leq Z_{\beta} \tilde{Z}_{\alpha}\left|\sigma_{\beta} \tilde{\sigma}_{\alpha}\right| \cdot \Delta t \cdot\left\{-\left(c_{1}\left|\tilde{p}_{l}-1\right|+c_{2} h_{r}\right)+\left|\tilde{p}_{l}-1\right| h_{r}\left(c_{1} c_{2} \Delta t+\mathcal{O}(1)\right)\right\} .
$$

Note that the positive terms are multiplied by $h_{r}$, while the leading negative term is not. Hence, by choosing $\|h\|_{\mathbf{L}^{\infty}}$ and $\Delta t$ small enough, we conclude that $\widetilde{\mathcal{Q}}$ decreases.

Case 4. We now consider the case of two non-approaching waves at $t_{k-1}$, a 1-wave of strength $\sigma_{\alpha}$ at $x_{\alpha}$ and a 2-wave of strength $\tilde{\sigma}_{\beta}$ at $\tilde{x}_{\beta}$, with $x_{\alpha}<\tilde{x}_{\beta}$. At time $t_{k}$, after the fractional step (3.2), each wave will become two waves, one from each family, with strengths $\left(\sigma_{\alpha}^{+}, \sigma_{\beta}^{+}\right)$and $\left(\tilde{\sigma}_{\alpha}^{+}, \tilde{\sigma}_{\beta}^{+}\right)$located at $x_{\alpha}^{+}$and $\tilde{x}_{\beta}^{+}$, respectively. See figure 6 for an illustration.

Since the two waves are not approaching at $t_{k-1}$, we have $\widetilde{\mathcal{Q}}\left(t_{k-1}\right)=0$. But at $t_{k}, \widetilde{\mathcal{Q}}$ will no longer be zero. Using (3.4) and (3.5), the increments can be estimated as

$$
\Delta \widetilde{\mathcal{Q}}_{h h}=w_{\alpha^{+}, \tilde{\alpha}^{+}} \cdot Z_{\alpha}^{+}\left|\sigma_{\alpha}^{+}\right| \cdot \tilde{Z}_{\beta}^{+}\left|\tilde{\sigma}_{\alpha}^{+}\right| \leq \mathcal{O}(1) \cdot Z_{\alpha}\left|\sigma_{\alpha}\right| \cdot \tilde{Z}_{\beta}\left|\tilde{\sigma}_{\beta}\right| \cdot \tilde{h}_{l} \cdot \Delta t
$$

and

$$
\Delta \widetilde{\mathcal{Q}}_{p h}+\Delta \widetilde{\mathcal{Q}}_{p p}=Z_{\alpha}^{+}\left|\sigma_{\beta}^{+}\right| \cdot \tilde{Z}_{\beta}^{+}\left(\left|\tilde{\sigma}_{\alpha}^{+}\right|+\left|\tilde{\sigma}_{\beta}^{+}\right|\right) \leq \mathcal{O}(1) \cdot Z_{\alpha}\left|\sigma_{\alpha}\right| \cdot \tilde{Z}_{\beta}\left|\tilde{\sigma}_{\beta}\right| \cdot\left|p_{l}-1\right| \cdot \Delta t .
$$

Adding up these two estimates, we get the estimate for $\widetilde{\mathcal{Q}}$

$$
\Delta \widetilde{\mathcal{Q}}=\mathcal{O}(1) \cdot Z_{\alpha}\left|\sigma_{\alpha}\right| \cdot \tilde{Z}_{\beta}\left|\tilde{\sigma}_{\beta}\right| \cdot\left(\left|p_{l}-1\right|+\tilde{h}_{l}\right) \cdot \Delta t .
$$




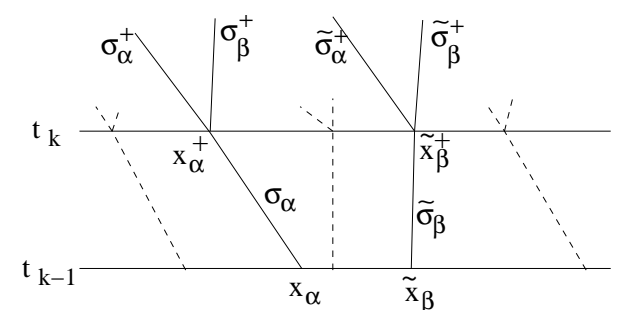

Figure 6: Case 4, two non-approaching waves, a 1-wave $\sigma_{\alpha}$ and a 2 -wave $\tilde{\sigma}_{\beta}$ at $t_{k-1}$. Here the dotted lines denote other wave fronts which are not being considered in this case.

The general case. In the general case, we sum up all the possible contributions due to each pair of waves and get

$$
\begin{aligned}
\Delta \widetilde{\mathcal{Q}} \leq & \mathcal{O}(1) \Delta t \sum_{i_{\alpha}=i_{\gamma}=1, x_{\alpha}<x_{\gamma}} Z_{\alpha} Z_{\gamma}\left|\sigma_{\alpha} \sigma_{\gamma}\right| \cdot\left|P_{l}^{\alpha}-1\right|+ \\
& +\mathcal{O}(1) \Delta t \sum_{i_{\alpha}=1, i_{\beta}=2, x_{\alpha}<x_{\beta}} Z_{\alpha} Z_{\beta}\left|\sigma_{\alpha} \sigma_{\beta}\right| \cdot\left(\left|p_{l}^{\alpha}-1\right|+h_{l}^{\beta}\right) \\
\leq & \mathcal{O}(1) \Delta t\left\{\sum_{i_{\alpha}=1} Z_{\alpha}\left|\sigma_{\alpha}\right|\left|p_{l}^{\alpha}-1\right|+\sum_{i_{\beta}=2} Z_{\beta}\left|\sigma_{\beta}\right| h_{l}^{\beta}\right\} \cdot \widetilde{V}\left(t_{k-1}\right) .
\end{aligned}
$$

\subsubsection{The decrease of $\widetilde{\mathcal{S}}$}

Finally, the change of $\widetilde{\mathcal{S}}=\widetilde{V}+c \widetilde{\mathcal{Q}}$ follows from (4.46) and (4.49). We have

$$
\begin{aligned}
\Delta \widetilde{V}+c \Delta \widetilde{\mathcal{Q}} \leq & \Delta t \cdot \sum_{i_{\alpha}=1} Z_{\alpha}\left|\sigma_{\alpha}\right| \cdot\left|p_{l}^{\alpha}-1\right|\left(-c_{1}+\mathcal{O}(1) \cdot c \widetilde{V}\left(t_{k-1}\right)\right) \\
& +\Delta t \cdot \sum_{i_{\beta}=2} Z_{\beta}\left|\sigma_{\beta}\right| h_{l}^{\beta}\left(-c_{2}+\mathcal{O}(1) \cdot c \widetilde{V}\left(t_{k-1}\right)\right)
\end{aligned}
$$

hence $\Delta \widetilde{\mathcal{S}} \leq 0$ provided that $c$ is small enough comparing to $c_{1}$ and $c_{2}$.

In summary, we see that by our choice of the weights $Z(t, x)$, the functional $\widetilde{\mathcal{S}}$ is nonincreasing from time $t_{k-1}+$ to $t_{k}+$, because the possible increase caused by the source term is counter balanced by the decrease in the weight $Z$. In addition, from the analysis in Section 4.4 we see that $\widetilde{\mathcal{S}}$ decreases strictly at every interaction. Since $\widetilde{\mathcal{S}}$ is bounded and positive, then the total decrease of $\widetilde{\mathcal{S}}$ caused by all interactions (through all time $t>0$ ) is bounded. In term, this gives a bound on the total amount of interaction and cancellation through all time $t>0$.

Remark. We remark that one can actually choose $c=1$ in the definition of $\widetilde{\mathcal{S}}$. In fact, the constants $c_{1}$ and $c_{2}$ can be sufficiently large by choosing $\kappa_{1}$ and $\kappa_{2}$ large, such that one still achieves $\Delta \widetilde{\mathcal{S}} \leq 0$ in (4.50). We kept the constant $c$ since this keeps our argument more straight forward. 


\section{Global existence of BV solutions; Putting things together to complete the proof of Theorem 1}

In this section we fill in some remaining details in the previous analysis in Section 4, and achieve a rigorous proof of Theorem 1 .

As remarked at the beginning of Section 4, the approximate solutions are defined by the front tracking approximation, as in $[8,9,4]$ on each time interval $\left[t_{k-1}, t_{k}\right)$, and then updating the solution at time $t=t_{k}$ according to (3.2). In general, for $2 \times 2$ systems the presence of non-physical fronts can be avoided completely [4, 9]. Furthermore, it is important to observe that error terms of the form

$$
\mathcal{O}(1) \cdot[\text { maximum strength of rarefaction fronts }]
$$

can be neglected. Finally, error terms of the form

$$
\mathcal{O}(1) \cdot \Delta t \cdot[\text { total amount of interaction }+ \text { cancellation }]
$$

can also be neglected, because they become vanishingly small as $\Delta t=t_{k}-t_{k-1}$ approaches zero.

We now review the steps in the previous section, and check that all the estimates are valid, up to vanishingly small error terms as in (5.1)-(5.2).

1. During the time interval $\left(t_{k-1}, t_{k}\right)$, the quantities $\int h d x$ and $\int(p-1) d x$ are conserved by front tracking approximations, except for errors of the order (5.1).

The quantity $\int|p-1| d x$ may increase only in the case of the rarefaction waves of the second family where $p-1$ changes sign. However, one can easily check that the possible increase is bounded by a quantity of the order (5.1).

On the other hand, the difference $\mathcal{I}^{h}\left(t_{k}\right)-\mathcal{I}^{h}\left(t_{k-1}\right)$ can be bounded as in (4.8), plus error terms of size

$\mathcal{O}(1) \cdot \Delta t \cdot\left[\right.$ amount of interaction and cancellation during the interval $\left.\left[t_{k-1}, t_{k}\right)\right]$.

Indeed, for the piecewise constant approximate solution, during $\left[t_{k-1}, t_{k}\right)$ we have

$$
(W h)_{t}-(W h(q+1))_{x} \leq-W h(h+q+1) \cdot|q| .
$$

Therefore $\mathcal{I}^{h}$ will decrease on this interval,

$$
\mathcal{I}^{h}\left(t_{k}-\right)-\mathcal{I}^{h}\left(t_{k-1}\right) \leq-\int_{t_{k-1}}^{t_{k}} \int_{-\infty}^{\infty} W h(h+q+1) \cdot|q| d x d t .
$$

At the time step (3.2) when the source term is added, $\mathcal{I}^{h}$ may increase, as

$$
\mathcal{I}^{h}\left(t_{k}\right)-\mathcal{I}^{h}\left(t_{k}-\right)=\Delta t \int_{-\infty}^{\infty} W q h\left(x, t_{k}-\right) d x .
$$

Adding these two estimates, one gets

$$
\begin{aligned}
\mathcal{I}^{h}\left(t_{k}\right)-\mathcal{I}^{h}\left(t_{k-1}\right) \leq & \int_{t_{k-1}}^{t_{k}} \int_{-\infty}^{\infty} W h(q-|q|(h+q+1)) d x d t \\
& +\left[\Delta t \int_{-\infty}^{\infty} W q h\left(x, t_{k}-\right) d x-\int_{t_{k-1}}^{t_{k}} \int_{-\infty}^{\infty} W q h(x, t) d x d t\right] .
\end{aligned}
$$


Here the first term on the right-hand side is negative, and the second term is bounded by (5.3). As remarked earlier, these are vanishingly small, as $\Delta t \rightarrow 0$.

2. Concerning the quantity $P_{\text {inf }}$ for the approximate solution, it might decrease at an interaction and at where a Riemann problem is solved. We now give a rigorous analysis.

First, at the initial time $t=0+$, Riemann problems are solved and $P_{\text {inf }}$ might decrease, but by a small amount. In fact, let $\left(H_{l}, P_{l}\right),\left(H_{r}, P_{r}\right)$ be the left and right states of a Riemann problem, and $\left(H_{m}, P_{m}\right)$ be the middle state. $P_{m}$ may be smaller than $P_{l}$ if the 1-wave is a shock. In this case, let $\sigma_{h}$ be the strength of the 1-wave. Recalling (3.23), we have

$$
\left|P_{m}-P_{l}\right|=\mathcal{O}(1) \sigma_{h}^{2}\left|P_{l}-1\right|=\mathcal{O}(1)\|h\|_{\mathbf{L}^{\infty}}^{2} .
$$

Then, the possible decrease of $P_{\text {inf }}$ at $t=0+$ is very small.

Second, during the time interval $\left(t_{k-1}, t_{k}\right), P_{\text {inf }}$ might decrease at some interaction points. This could happen if the outgoing 1-wave $\sigma_{h}$ is a shock. We claim that the possible total decrease in $P_{\text {inf }}$ caused by interaction through all time $t>0$ is of order

$$
\mathcal{O}(1) \cdot\|h\|_{\mathbf{L}^{\infty}} \cdot[\text { total amount of interaction }+ \text { cancellation }] .
$$

Indeed, consider an interaction between two waves $\sigma_{\alpha}$ and $\sigma_{\beta}$, and use the notation of Sect. 4.4. Let $\left(H_{l}, P_{l}\right),\left(H_{m}, P_{m}\right)$ and $\left(H_{r}, P_{r}\right)$ be the left, middle and right state of the outgoing waves. We consider 4 cases listed in Sect. 4.4.

(1). Two neighboring $h$-shocks with strength $\sigma_{\alpha}$ and $\sigma_{\beta}$ interact. In this case, the change in $\widetilde{\mathcal{S}}$ caused by the interaction is

$$
|\Delta \widetilde{\mathcal{S}}|=\mathcal{O}(1) w_{\alpha, \beta} \cdot\left|\sigma_{\alpha} \sigma_{\beta}\right|
$$

Then we have

$$
\left|P_{m}-P_{r}\right|=\left|\sigma_{p}^{\prime}\right|=\mathcal{O}(1) \cdot \frac{w_{\alpha, \beta}}{\delta_{0}} \cdot\|h\|_{\mathbf{L}^{\infty}} \cdot\left|\sigma_{\alpha} \sigma_{\beta}\right|=\mathcal{O}(1) \cdot \frac{\|h\|_{\mathbf{L}^{\infty}}}{\delta_{0}} \cdot|\Delta \widetilde{\mathcal{S}}| .
$$

(2). Two $h$-waves of opposite sign interact. Here one has $|\Delta \widetilde{\mathcal{S}}|=\mathcal{O}(1) \min \left\{\left|\sigma_{\alpha}\right|,\left|\sigma_{\beta}\right|\right\}$ and

$$
\left|P_{m}-P_{r}\right|=\left|\sigma_{p}^{\prime}\right|=\mathcal{O}(1) \cdot\|h\|_{\mathbf{L}^{\infty}} \cdot\left|\sigma_{\alpha} \sigma_{\beta}\right| \leq \mathcal{O}(1) \cdot\|h\|_{\mathbf{L}^{\infty}} \cdot|\Delta \widetilde{\mathcal{S}}|
$$

(3). A $p$-wave $\sigma_{\alpha}$ interacts with an $h$-wave $\sigma_{\beta}$. In this case, $|\Delta \widetilde{\mathcal{S}}|=\mathcal{O}(1)\left|\sigma_{\alpha} \sigma_{\beta}\right|$. Let $P_{*}$ be the intermediate value before interaction, then

$$
\left|P_{m}-P_{l}\right|=\mathcal{O}(1)\left|P_{*}-P_{r}\right| \cdot\left|\sigma_{\alpha}\right|=\mathcal{O}(1)\left|P_{*}-1\right| \cdot\left|\sigma_{\beta}\right|^{2} \cdot\left|\sigma_{\alpha}\right|=\mathcal{O}(1) \cdot\|h\|_{\mathbf{L}^{\infty}} \cdot|\Delta \widetilde{\mathcal{S}}| .
$$

(4). Two $p$-waves interact. Here we have again $|\Delta \widetilde{\mathcal{S}}|=\mathcal{O}(1)\left|\sigma_{\alpha} \sigma_{\beta}\right|$ and

$$
\left|P_{m}-P_{l}\right|=\mathcal{O}(1)\left(\sigma_{h}^{\prime}\right)^{2}=\mathcal{O}(1)\left(\|h\|_{\mathbf{L}^{\infty}}\left|\sigma_{\alpha} \sigma_{\beta}\right|\right)^{2} \leq \mathcal{O}(1)\|h\|_{\mathbf{L}^{\infty}}|\Delta \widetilde{\mathcal{S}}|
$$


We see in all cases, the possible decrease of $P$ is of the order

$$
\mathcal{O}(1)\|h\|_{\mathbf{L}^{\infty}}\left(1+\frac{1}{\delta_{0}}\right)|\Delta \widetilde{\mathcal{S}}|
$$

where $|\Delta \widetilde{\mathcal{S}}|$ is the change in $\widetilde{\mathcal{S}}$ due to the interaction. Since $\widetilde{\mathcal{S}}$ is strictly decreasing at every interaction, and the total decrease in $\widetilde{\mathcal{S}}$ caused by all interaction is bounded, we see that the possible total decrease in $P_{\text {inf }}$ through all time $t>0$ caused by interaction is of order $\mathcal{O}(1)\|h\|_{\mathbf{L}^{\infty}}\left(1+\frac{1}{\delta_{0}}\right) \widetilde{\mathcal{S}}(0)$, which is as small as we like.

Finally, we analyze the source step (3.2). From the formal argument in Section 4.2, we see that the source term "helps" keeping $P$ bounded away from 0 . Now we check if a similar situation happens in the discrete approximate solutions.

We note that $P_{\mathrm{inf}} \leq 1$, which follows from the bound on $\|P-1\|_{\mathbf{L}^{1}}$. Since we are interested in a lower bound on $P$ of the form $P \geq P_{0}>0$, we focus our attention on the case where $P<1$, say $P \leq 0.5$ and $p \leq 0.5$.

We claim that at each source step (3.2), $P_{\text {inf }}$ will increase. Indeed, if $p<1(P<1)$, we see $h$ will decrease. From the geometry of the characteristics in Fig. 1, we see that $P$ will increase.

However, when Riemann problems are solved right after (3.2), $P_{\text {inf }}$ might decrease if the outgoing 1-wave is a shock. We claim that the possible decrease of $P_{\text {inf }}$ caused by the Riemann problem is counter balanced by the increase of $P_{\text {inf }}$ in the source step (3.2), so $P_{\text {inf }}$ will not decrease from $t_{k}-$ to $t_{k}+$.

Let the capital letters $(H, P)$ denote the Riemann coordinate of $(h, p)$. Let $\left(H_{l}, P_{l}\right)\left(H_{r}, P_{r}\right)$ and $\left(H_{l}^{+}, P_{l}^{+}\right),\left(H_{r}^{+}, P_{r}^{+}\right)$be the left, right states of the incoming and outgoing waves, respectively, and $\left(H_{m}^{+}, P_{m}^{+}\right)$be the middle state of the outgoing waves. We first consider the case of a incoming 1-wave. This must be of the same type as the outgoing 1-wave, which is a shock. Then, $h_{l}^{+} \leq h_{l}$ and $p_{l}=p_{l}^{+}$, so the 1-shock curve from $\left(h_{l}^{+}, p_{l}^{+}\right)$will lie above the one from $\left(h_{l}, p_{l}\right)$. Also, $h_{r}^{+}<h_{r}$, because the 2-curves have negative slope in $(h, p)$-plane. From the geometry of the shock and rarefaction curves, and the uniqueness of the 1-shock and 1-rarefaction curves, we must have $H_{l}^{+}<H_{m}^{+}<H_{r}$. By the uniqueness of 1-shock and 1-characteristic curves, $P_{m}^{+}$ must lie between $P_{l}$ and $P_{l}^{+}$. Therefore $P_{\text {inf }}$ will not decrease.

Secondly, we consider an incoming 2 -wave of strength $\sigma_{p}$ with $p_{l} \leq 0.5$ and $h_{l}>0$. Then, $P_{l}^{+}>P_{l}$, and we have the following estimate on the increment

$$
P_{l}^{+}-P_{l} \geq \bar{c} \Delta t h_{l},
$$

for some constant $\bar{c}>0$. By the estimate (3.5) the strength of the outgoing 1-wave is $\sigma_{h}^{\prime}=$ $\mathcal{O}(1) \Delta t h_{l}\left|\sigma_{p}\right|$. Then, by (3.23) the difference between $P_{m}^{+}$and $P_{l}^{+}$is of order $\mathcal{O}(1)\left(\sigma_{h}^{\prime}\right)^{2}\left(1-p_{l}^{+}\right)$, so we have

$$
\left|P_{l}^{+}-P_{m}^{+}\right|=\mathcal{O}(1)\left(\Delta t h_{l}\right)^{2}\left(\sigma_{p}\right)^{2}\left(1-p_{l}^{+}\right) .
$$

Here the terms $\sigma_{p}$ and $\left(1-p_{l}^{+}\right)$are bounded. By choosing $\|h\|_{\mathbf{L}^{\infty}}$ and $\Delta t$ sufficiently small, the possible decrease will be much smaller than the increment. Therefore $P_{\text {inf }}$ will not decrease.

This completes the uniform lower bound on $P$ (or equivalently, on $p$ ).

3. Concerning the functionals $\mathcal{J}^{h}$, and $\mathcal{J}^{p}$ during each interval $\left[t_{k-1}, t_{k}\right]$ their sizes may increase, but only in the amounts that are vanishingly small. Indeed, this can be achieved by a similar argument as for $\mathcal{I}^{h}$. We will work out the details for the quantity $\mathcal{J}^{p}$, and omit the details for 
$\mathcal{J}^{h}$ which is similar. On the interval $\left[t_{k-1}, t_{k}\right)$, consider a 2-characteristic $t \mapsto x_{2}(t)$. Then $P$ remains constant along this curve unless it crosses a 1-shock. If this happens, $P$ will change by a small amount due to the second order tangency of shock and rarefaction curves. Let $\sigma_{h}$ be the strength of the 1 -shock, and $P^{+}$be the value of $P$ after crossing this shock; again by (3.23), we have

$$
P^{+}-P=\mathcal{O}(1) \sigma_{h}^{2}=\mathcal{O}(1)\|h\|_{\mathbf{L}^{\infty}}^{2},
$$

which shows that the change in $P$ is vanishingly small.

Now, ignore interactions and the crossings of 1-waves, then $P$ remains constant along 2characteristic $t \mapsto x_{2}(t)$, but $V^{p}$ will decrease. We have

$$
\begin{aligned}
\frac{d}{d t} V^{p}\left(t, x_{2}(t)\right) & =\kappa_{2} V^{p}\left(-W h \dot{x}_{2}+\int_{x_{2}(t)}^{\infty}(W h(y, t))_{t} d y\right) \\
& \leq-\kappa_{2} V^{p}\left(W h\left(\dot{x}_{2}+q+1\right)+\int_{x_{2}(t)}^{\infty} W h|q|(h+q+1) d y\right)
\end{aligned}
$$

which gives

$$
\begin{aligned}
V^{p} P\left(t_{k}-\right. & \left., x_{2}\left(t_{k}-\right)\right)-V^{p} P\left(t_{k-1}, x_{2}\left(t_{k-1}\right)\right) \\
& \leq-\int_{t_{k-1}}^{t_{k}} \kappa_{2} V^{p} P\left(W h\left(\dot{x}_{2}+q+1\right)+\int_{x_{2}(t)}^{\infty} W h|q|(h+q+1) d y\right) d t .
\end{aligned}
$$

At $t_{k}$ when the step (3.2) is performed, both $V^{p}$ and $P$ will change. Calling $P^{+} \doteq P\left(t_{k}, x_{2}\left(t_{k}\right)\right)$ and $P \doteq P\left(t_{k}-, x_{2}\left(t_{k}-\right)\right)$, we have

$$
P^{+} \leq P+\mathcal{O}(1) \Delta t\left|\frac{\partial P}{\partial h}\right| \cdot|q| h=P \cdot\left(1+\mathcal{O}(1) \Delta t\left|\frac{\partial P}{\partial h}\right| \frac{|q| h}{P}\right) \leq P \cdot\left(1+\mathcal{O}(1) \Delta t\left|\frac{\partial P}{\partial h}\right| \cdot|q| h\right)
$$

where the last inequality holds because $P$ is bounded away from 0 . Therefore we get

$$
\begin{aligned}
V^{p+} P^{+} & \leq \exp \left(\kappa_{2} \int_{x_{2}\left(t_{k}\right)}^{\infty} W(h+\Delta t q h) d y\right) \cdot P \cdot\left(1+\mathcal{O}(1) \Delta t\left|\frac{\partial P}{\partial h}\right||q| h\right) \\
& =V^{p} P \cdot \exp \left(\kappa_{2} \Delta t \int_{x_{2}\left(t_{k}\right)}^{\infty} W q h d y\right) \cdot\left(1+\mathcal{O}(1) \Delta t\left|\frac{\partial P}{\partial h}\right||q| h\right) \\
& \leq V^{p} P \cdot\left(1+\kappa_{2} \Delta t \int_{x_{2}\left(t_{k}\right)}^{\infty} W q h d y+\mathcal{O}(1) \Delta t^{2}\|h\|_{\mathbf{L}^{\infty}}^{2}\right) \cdot\left(1+\mathcal{O}(1) \Delta t\left|\frac{\partial P}{\partial h}\right||q| h\right) \\
& =V^{p} P\left[1+\kappa_{2} \Delta t \int_{x_{2}\left(t_{k}\right)}^{\infty} W h q d y+\mathcal{O}(1) \Delta t\left|\frac{\partial P}{\partial h}\right||q| h+\mathcal{O}(1) \Delta t^{2}\|h\|_{\mathbf{L}^{\infty}}^{2}\right] .
\end{aligned}
$$

Here for the second last inequality we used $e^{x}=1+x+\mathcal{O}(1) x^{2}$ for small $x$, and in the last equality we gather all the high order terms in the last term. Adding up this inequality with 
(5.5) and rearranging the terms, we get

$$
\begin{aligned}
& V^{p} P\left(t_{k}, x_{2}\left(t_{k}\right)\right)-V^{p} P\left(t_{k-1}, x_{2}\left(t_{k-1}\right)\right) \\
& \leq-\kappa_{2} \int_{t_{k-1}}^{t_{k}} V^{p} P \int_{x_{2}(t)}^{\infty} W h[|q|(h+q+1)-q] d y d t \\
&+\kappa_{2} \Delta t V^{p} P\left(t_{k}\right) \int_{x_{2}\left(t_{k}\right)}^{\infty} W h q d y-\kappa_{2} \int_{t_{k-1}}^{t_{k}} V^{p} P(t) \int_{x_{2}(t)}^{\infty} W h q d y d t \\
&-\int_{t_{k-1}}^{t_{k}} V^{p} P\left[\kappa_{2} W h\left(\dot{x}_{2}+q+1\right)-\mathcal{O}(1)\left|\frac{\partial P}{\partial h}\right||q| h\right] d t \\
&+\mathcal{O}(1)\left[\Delta t V^{p} P\left|\frac{\partial P}{\partial h}\right||q| h\left(t_{k}\right)-\int_{t_{k-1}}^{t_{k}} V^{p} P\left|\frac{\partial P}{\partial h}\right||q| h(t) d t\right] \\
&+\mathcal{O}(1) \Delta t^{2}\|h\|_{\mathbf{L}^{\infty}}^{2} .
\end{aligned}
$$

Here the term (5.6) is negative, and (5.8) is also negative by choosing $\kappa_{2}$ sufficiently large. However all the other terms might be positive, but they are small. In fact, (5.7) is bounded by

$\kappa_{2} \mathcal{O}(1) \Delta t\|h\|_{\mathbf{L}^{\infty}} \cdot\left[\right.$ amount of interaction and cancellation during the interval $\left.\left[t_{k-1}, t_{k}\right)\right]$,

and (5.9) is bounded by

$$
\mathcal{O}(1) \Delta t\|h\|_{\mathbf{L}^{\infty}} \cdot\left[\text { amount of interaction and cancellation during the interval }\left[t_{k-1}, t_{k}\right)\right] \text {. }
$$

Finally, since (5.10) is vanishingly small, we conclude that $\mathcal{J}^{p}$ is non-increasing up to a vanishingly small error, achieving the uniform bound on $P$.

4. The order of the constants should be chosen as follows. After the constants $M$ and $p_{0}$ have been given, we can choose $\delta_{0}>0$ so that $\Delta \mathcal{S} \leq 0$ in section 4.4. Then we choose $\kappa_{1}$ large enough so that (4.42) holds, then $\kappa_{2}$ large enough such that (4.45) holds, and then $c$ sufficiently small in subsection 4.6.3 such that $\Delta \widetilde{\mathcal{S}} \leq 0$.

Finally we choose $\delta=\|\bar{h}\|_{\mathbf{L}^{\infty}}$ sufficiently small. This will render $\mathcal{J}^{h}(t)$ as small as required, for all times $t \geq 0$.

Moreover, at each time $t_{k}$, the inequalities (4.17)-(4.18) hold, up to error terms of magnitude (5.3). These are vanishingly small as $\Delta t \rightarrow 0$.

5. Concerning the total variation, the decrease of the functional $\widetilde{\mathcal{S}}$ in (4.34) during the intervals $\left[t_{k-1}, t_{k}\right]$ has already been analyzed in the previous section, without taking into consideration of the interactions. Adding the effect of interactions will only introduce small error terms as in (5.1)-(5.2), which can be arbitrary small. Therefore, the estimates (4.42) and (4.45) will still be valid. This completes the proof of Theorem 1.

\section{Concluding Remarks}

(1). In this paper we proved the global existence of large BV solutions to a $2 \times 2$ system of balance laws in one space dimension, arising from a model of granular flow. However, we left open the question of uniqueness of entropy-weak solutions. This does not immediately follow 
from the known results [15], because one of the characteristic fields is neither genuinely nonlinear, nor linearly degenerate. Uniqueness and continuous dependence of large BV solutions may be obtained by putting together the techniques in [4] and [15]. Alternatively, it may be achieved by further developing the vanishing viscosity approach in $[7,12]$.

(2). A related interesting problem is the slow erosion limit, when the thickness of the moving layer is very small. In [26] we observed from numerical simulations that, as long as the moving layer remains very thin, the evolution of the standing profile depends only on the total amount of sand being poured from the top, not on the specific rate at which the sand is poured. In a forthcoming paper [3], this observation is studied in detail. We prove that in the limit as the thickness of the moving layer $h$ tends to zero, the evolution of the standing profile can be described by a scalar integro-differential equation for the slope $p=u_{x}$.

(3). It is also of great interest to study the case where the slope $p=u_{x}$ of the standing layer changes sign. This appears to be an essential step also toward the study of the two-dimensional case, since the directional derivative of $u$ generically changes sign along a straight line.

Unfortunately, as $p$ changes sign, the flux function is no longer smooth, but only Lipschitz continuous. This does not fit in the standard theory of hyperbolic conservation laws. However, the Riemann problem is still well-defined and can be solved, possibly leading to some results on the Cauchy problem.

Acknowledgement. The authors are grateful to Prof. Alberto Bressan for suggesting the problem and for many useful discussions. We also thank the reviewer for carefully reading our manuscript.

\section{References}

[1] Amadori, D. and Gosse, L. and Guerra, G.; Global BV entropy solutions and uniqueness for hyperbolic systems of balance laws. Arch. Rational Mech. Anal. 162 (2002), 327-366.

[2] Amadori, D. and Guerra, G.; Uniqueness and continuous dependence for systems of balance laws with dissipation. Nonlinear Analysis TMA 49 (2002), 987-1014.

[3] Amadori, D. and Shen, W.; The Slow Erosion Limit for a Model of Granular Flow, Preprint 2008 .

[4] Ancona, F. and Marson, A.; Well-posedness for general $2 \times 2$ systems of conservation laws. Mem. Amer. Math. Soc. 169 (2004), no. 801.

[5] Ancona, F. and Marson, A.; Existence Theory by Front Tracking for General Nonlinear Hyperbolic Systems. Arch. Rational Mech. Anal. 185 (2007), 287-340.

[6] Boutreux, T. and Gennes, P.-G.; Surface flows of granular mixtures, I. General principles and minimal model. J. Phys. I France 6 (1996), 1295-1304.

[7] Bianchini, S. and Bressan, A.; Vanishing viscosity solutions to nonlinear hyperbolic systems. Annals of Mathematics 161 (2005), 223-342. 
[8] Bressan, A.; Hyperbolic Systems of Conservation Laws. The One Dimensional Cauchy Problem, Oxford University Press, 2000.

[9] Bressan, A. and Colombo, R.M.; The semigroup generated by $2 \times 2$ conservation laws, Arch. rational Meth. Anal. textbf133(1995), no. 1, 1-75.

[10] Cannarsa, P. and Cardaliaguet, P.; Representation of equilibrium solutions to the table problem for growing sandpiles. J. Eur. Math. Soc. (JEMS) 6 (2004), 435-464.

[11] Cannarsa, P. and Cardaliaguet, P. and Crasta, G. and Giorgieri, E.; A boundary value problem for a PDE model in mass transfer theory: representation of solutions and applications. Calc. Var. Partial Differential Equations 24 (2005), 431-457.

[12] Christoforou, C.; Hyperbolic systems of balance laws via vanishing viscosity. J. Differential Equations 221 (2006), 470-541.

[13] Christoforou, C.; Uniqueness and sharp estimates on solutions to hyperbolic systems with dissipative source. Comm. P.D.E. 31 (2006), 1825-1839.

[14] Colombo, R.M. and Corli, A.; On a class of hyperbolic balance laws. J. Hyperbolic Differ. Equ. 1 (2004), no. 4, 725-745.

[15] Crasta, G. and Piccoli, B.; Viscosity solutions and uniqueness for systems of inhomogeneous balance laws. Discrete Contin. Dynam. Systems 3 (1997), 477-502.

[16] Dafermos, C.M. and Hsiao, L.; Hyperbolic systems of balance laws with inhomogeneity and dissipation. Indiana U. Math. J. 31 (1982), 471-491.

[17] Duran, J.; Sands, Powders, and Grains: An Introduction to the Physics of Granular Materials, Springer-Verlag, 2000.

[18] Glimm, J.; Solutions in the large for nonlinear hyperbolic systems of equations. Comm. Pure Appl. Math. 18 (1965), 697-715.

[19] Glimm, J. and Lax, P.; Decay of solutions of systems of nonlinear hyperbolic conservation laws. Mem. Amer. Math. Soc. 101 (1970).

[20] Hadeler, K.P. and Kuttler, C.; Dynamical models for granular matter. Granular Matter 2 (1999), 9-18.

[21] Liu, T.P.; Admissible solutions of hyperbolic conservation laws. Mem. Amer. Math. Soc. 30 (1981), no. 240.

[22] Liu, T.P.; Quasilinear hyperbolic systems. Commun. Math. Phys. 68 (1979), 141-172.

[23] Liu, T.P. and Yang, T.; Weak Solutions of General Systems of Hyperbolic Conservation Laws. Comm. Math. Phys. 230 (2002), 289-327.

[24] Nishida, T. and Smoller, J.; Solutions in the large for some nonlinear conservation laws. Comm. Pure Appl. Math. 26 (1973), 183-200.

[25] Serre, D.; Systems of Conservation Laws I, II, Cambridge University Press, 2000. 
[26] Shen, W.; On the Shape of Avalanches. J. Math. Anal. Appl. 339 (2008), 828-838.

[27] Smoller, J.; Shock Waves and Reaction-Diffusion Equations, Springer-Verlag, 1983. 